\title{
Low-frequency vibrations of two-dimensional droplets on heterogeneous substrates
}

\author{
Nikos Savva ${ }^{1,2}$ and Serafim Kalliadasis ${ }^{2,} \dagger$ \\ ${ }^{1}$ School of Mathematics, Cardiff University, Cardiff CF24 4AG, UK \\ ${ }^{2}$ Department of Chemical Engineering, Imperial College London, London SW7 2AZ, UK
}

(Received 6 November 2013; revised 15 April 2014; accepted 14 July 2014)

We present a theoretical investigation of the effects of low-frequency vibrations on the motion of two-dimensional droplets on heterogeneous substrates in the presence of gravity and substrate heterogeneities, both chemical and topographical. A combined analytical and numerical approach is undertaken, extending the work of Savva \& Kalliadasis (J. Fluid Mech., vol. 725, 2013, pp. 462-491) on inclined heterogeneous substrates to include the effects of substrate vibrations. Via a matching procedure and under the quasi-static assumption, we obtain evolution equations for the moving fronts. These equations are then invoked in a wide variety of case studies. It is demonstrated that vertically vibrated horizontal ratcheted substrates can induce unidirectional motion. For inclined substrates, we focus on a number of qualitative aspects of the peculiar vibration-induced climbing of droplets reported in experiments by Brunet, Eggers \& Deegan (Phys. Rev. Lett., vol. 99, 2007, art. 144501). We examine the effects of weak inertia on the dynamics, deduce analytical criteria for the uphill motion in the limit of weak gravitational and vibrational effects, and demonstrate that substrate heterogeneities may be utilised to enhance droplet transport.

Key words: contact lines, drops, lubrication theory

\section{Introduction}

The dynamics of vibrating droplets is among the classical problems in fluid dynamics, dating back to the works of Kelvin (1863), Rayleigh (1879) and Lamb (1881), which investigated the vibration modes of free liquid drops. These studies have been extended to supported, nearly spherical, gravity-free droplets, both with pinned contact lines (see e.g. Strani \& Sabetta 1984, 1988; Celestini \& Kofman 2006; Bostwick \& Steen 2009; Vejrazka, Vobecka \& Tihon 2013) and movable contact lines with hysteresis (see e.g. Dong, Chaudhury \& Chaudhury 2006; Lyubimov, Lyubimova \& Shklyaev 2006; Fayzrakhmanova \& Straube 2009). In these settings, a common observation reported in experiments is that sufficiently strong vibrations excite waves on the droplet free surface, which may lead to fragmentation and spray formation if they become sufficiently violent (see e.g. James et al. 2003; Vukasinovic, Smith \& Glezer 2007, and references therein).

† Email address for correspondence: s.kalliadasis@imperial.ac.uk 
Droplet mobility in the presence of vibrations is also influenced by substrate heterogeneities. For instance, carefully fabricated heterogeneous substrates can be used to control the motion of droplets with the help of vibrations, which can be of interest in the design of microfluidic devices (see e.g. Daniel, Chaudhury \& de Gennes 2005; Duncombe et al. 2012a; Duncombe, Parsons \& Böhringer 2012b). Nevertheless, this problem has received much less attention theoretically, apart from a handful of studies that developed some phenomenological models to match the experimental observations.

In the seminal theoretical study of Johnson \& Dettre (1964) on the effects of substrate roughness on contact angle hysteresis, it was first suggested (but not studied in detail) that vibrations may be utilised as a means to help a droplet overcome the energy barriers associated with the heterogeneities (chemical and/or topographical) that pin the droplet on a substrate. The experiments by Smith \& Lindberg (1978) demonstrated this for the first time with acoustic vibrations imposed on horizontal substrates, where they observed that contact angle measurements varied with the levels of vibration.

Since then, a number of studies have been devoted to investigate how vibrations can alter the equilibrium state attained by the droplet, when vibrations cease. For example, Della Volpe et al. (2002) and Meiron, Marmur \& Saguy (2004) suggested that vibrations may be used to measure the so-called 'true' equilibrium contact angle at the global minimum of the free energy functional, which is attainable by escaping the local metastable states with the help of a sufficiently strong external forcing. In a more recent experimental study, where substrates were subjected to white noise vibrations, Mettu \& Chaudhury (2010) observed that the transition to this global equilibrium state occurs regardless of whether its initial state was advancing or receding, and attempted to rationalise their observations with a modified Langevin equation based on a phenomenological spring-type model. Noteworthy also are the experimental studies by Bormashenko et al. (2007a,b,c, 2008), where wetting transitions on artificial and natural surfaces were investigated in detail. They observed that, with the help of vibrations, droplets can move irreversibly from a state where air is trapped between the substrate asperities and the droplet, the so-called Cassie state, to the Wenzel state, where the fluid impregnates the asperities. According to Bormashenko et al. (2007c), this transition, which happens continuously rather than abruptly, appears to possess a distinct resonance character associated with the gravity-capillary waves induced on the free surface of a vibrated drop.

Substrate vibrations have also been studied with the aim of understanding how to control droplet mobility on surfaces. For example, Daniel \& Chaudhury (2002) and Daniel et al. $(2004,2005)$ studied extensively the influence of wettability gradients on vibrated horizontal substrates, observing that droplet vibrations can help a droplet to overcome the unavoidable substrate defects that tend to pin the contact line, to the point of observing enhanced unidirectional motion along the gradient. Their experiments with asymmetric vibrations (Daniel et al. 2005) also revealed the intricate interplay between droplet velocity, the characteristics of vibration, as well as the natural harmonics of the droplet, and pointed out the potential of utilising such mechanisms in batch microfluidic processes. More recently, Mettu \& Chaudhury (2011) focused on the role of hysteresis in the dynamics observed in Daniel et al. (2005) and argued that hysteresis can provide a symmetry-breaking mechanism to promote directed motion, provided that it is not sufficiently large to introduce a large pinning force that would prevent net transport. This prospect of utilising the combined effects of vibrations and heterogeneities as an efficient means to 
control droplet motion on horizontal substrates was further demonstrated in the experimental work of Duncombe and co-workers by using fabricated substrates with asymmetric topographical topographical (Duncombe et al. 2012a) and wettability features (Duncombe et al. 2012b).

Studies on the effect of vibrations on inclined substrates were first conducted by Brunet, Eggers \& Deegan (2007, 2009), in which they observed that vibrations are capable of inducing a counter-intuitive uphill motion against gravity. They attributed the observed dynamics to the breaking of symmetry due to the presence of the substrate. Noblin, Kofman \& Celestini (2009) investigated in further detail this symmetry breaking introduced through vibration by demonstrating experimentally that a combination of vertical and lateral vibrations on horizontal substrates can yield pronounced directed motion, by appropriately tuning the amplitude and phase difference between the two components of vibration.

The intriguing experiments by Brunet et al. (2007, 2009) also motivated a number of theoretical and numerical studies, which attempted to rationalise the phenomenon qualitatively by assuming thin two-dimensional (2D) drops (John \& Thiele 2010; Thiele \& John 2010; Benilov 2010, 2011; Benilov \& Billingham 2011), noting also the recent analyses on three-dimensional (3D) droplets reported by Benilov (2011) and Benilov \& Cummins (2013).

More specifically, John \& Thiele (2010) and Thiele \& John (2010) performed a series of numerical experiments using a hysteresis-free long-wave model in the Stokes regime by assuming a precursor film ahead of the moving fronts, and they demonstrated that uphill motion can occur by oblique vibrations in the low-frequency regime. They argued that the lateral oscillations of the droplet fronts exhibit net transport due to a nonlinear response to the changes in droplet mobility, which increases as the droplet thickens and decreases as the droplet flattens because of the normal component of vibration. This mechanism does not rely on the presence of hysteresis, since symmetry breaking is provided by the manner in which the substrate is vibrated.

The first analytical investigation of this problem was conducted by Benilov (2010) for $2 \mathrm{D}$ droplets in the quasi-static limit, by considering essentially the Young-Laplace equation supplemented by a condition at the moving front prescribing the contact line velocity as an arbitrary function of the static and dynamic apparent contact angles, concluding that uphill motion is impossible if the substrate is vibrated harmonically. In a subsequent study, Benilov (2011) revisited his work on 2D droplets and further extended it to a 3D geometry with small contact angles. For 2D droplets, he found that uphill motion is possible with harmonic vibrations if second-order effects are considered, but cannot occur at low frequencies. For 3D drops, he concluded that uphill motion is possible for both high and low frequencies if the vibrations become stronger than a certain threshold. In a more recent development, Benilov \& Cummins (2013) presented a similar type of analysis for 3D drops with large contact angles, obtaining the appropriate criteria for uphill motion to occur. Noteworthy also is the investigation of the inviscid limit of vibrated 2D drops by Benilov \& Billingham (2011), who developed a shallow-water model, imposing also the same type of speed-contact angle relations at the moving fronts as in the work of Benilov (2010, 2011). Their analysis considered perturbations from a steady state, studying the interaction of the spreading and swaying modes, which describe, respectively, the symmetric and antisymmetric deformations of the free surface of the droplet, both of which are prerequisite for uphill motion and maximise their effect when these modes interact in phase. 
The aim of the present study is to present a combined analytical and numerical investigation of the 2D droplet dynamics in the presence of substrate vibrations and heterogeneities, by extending the work of Savva \& Kalliadasis (2013, hereinafter referred to as SK) on inclined heterogeneous substrates to include the effects of substrate vibrations. We focus on a regime of low-frequency vibrations as done previously (see e.g. Benilov 2010, 2011; John \& Thiele 2010) and rigorously treat the problem in its quasi-static limit. We need to emphasise that our analysis differs from the general approach used by Benilov $(2010,2011)$ and Benilov \& Cummins (2013) in a number of respects. More specifically, in those studies the droplet shape was determined solely by balancing surface tension, gravitational and vibrational effects through the Young-Laplace equation, and driving the contact line dynamics by imposing a priori a speed-contact angle relation as a boundary condition. Here, instead, we consider the quasi-static dynamics of a time-dependent partial differential equation (PDE), which includes viscous effects under the long-wave assumption. Through the matched asymptotics that we employ, the speed-contact angle relation is eventually the product of asymptotic analysis. More importantly, this is the first study to treat the combined effects of vibration, gravity and substrate heterogeneities both analytically and numerically, allowing us to properly characterise the dynamics, without the need to utilise any of the ad hoc phenomenological spring-type models introduced in previous studies (see e.g. Daniel et al. 2005; Mettu \& Chaudhury 2010, 2011).

Our model, its main assumptions and boundary conditions are presented in $\S 2$. In $\S 3$ we derive evolution equations for the two moving fronts as a system of integro-differential equations (IDEs), by invoking the results in SK upon which the present study is based. In $\S 4$ we investigate the ideal-substrate case, which has been previously considered numerically by Thiele \& John (2010) using a precursor film model. Most notably, we derive the criteria for uphill droplet motion on ideal substrates and look into the influence of weak inertial effects, by investigating the corresponding Kármán-Pohlhausen model (see López, Miksis \& Bankoff 1997). In § 5 we consider some representative examples with heterogeneous substrates, discussing a number of interesting effects, such as the ability of heterogeneities to assist both the downhill and uphill motion. While more focus in this section is placed on inclined topographical substrates, some illustrative examples with horizontal and chemically heterogeneous substrates are also considered. Our discussion concludes in $\S 6$ with a summary and some final remarks.

\section{Model}

Consider a 2D droplet on the $X-Z$ plane, supported by a substrate that is inclined at angle $\phi$ to the horizontal and vibrated at angle $\psi$ with respect to the substrate vertical, experiencing an acceleration $\Gamma\left(T_{*}\right)$ at time $T_{*}$. The substrate is heterogeneous, with a topographical structure prescribed by a smooth profile $S(X)$, which is slowly varying with $X$, i.e. $\partial_{X} S \ll 1$. Moreover, the contact angle $\Theta(X)$, which is the angle between the tangent to the free surface of the droplet and the tangent to the substrate topography at the point of contact, varies locally according to $\tan \Theta=F(X)$, and is assumed to be small, i.e. $0<F(X) \ll 1$ (see figure 1 for a sketch of the geometry using dimensionless variables). As in SK, we assume slip along the substrate, so that

$$
\left.U\right|_{Z=S(X)}=\left.\Lambda \partial_{Z} U\right|_{Z=S(X)},
$$

where $U\left(X, Z, T_{*}\right)$ is the velocity in the $X$ direction and $\Lambda$ is the slip length, taken to be constant for simplicity. By neglecting fluid inertia (weak inertial effects are 


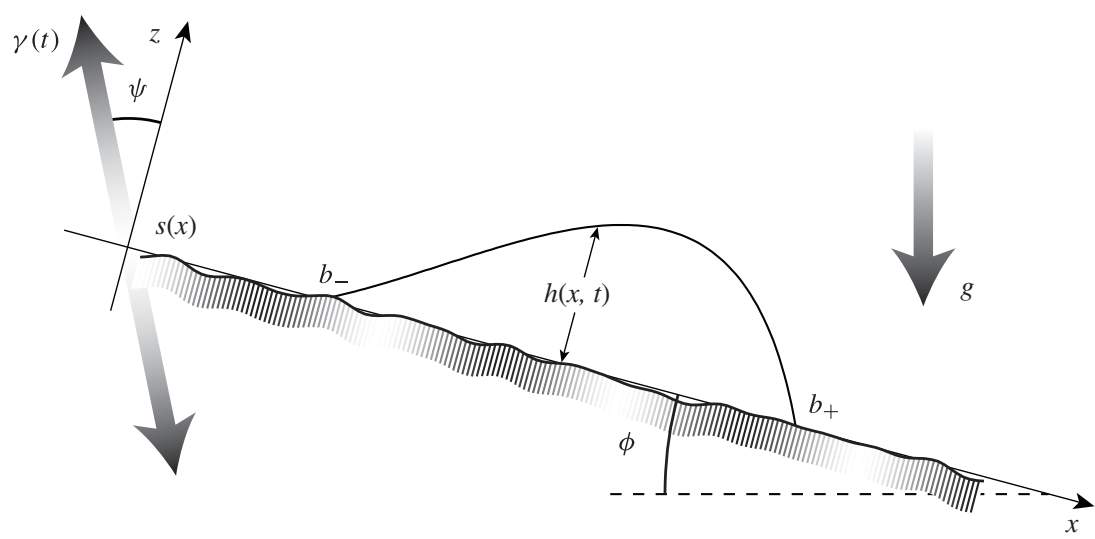

FIgURE 1. A 2D droplet on a heterogeneous substrate inclined at angle $\phi$ to the horizontal. In non-dimensional units, the droplet has thickness $h(x, t)$, with its contact points located at $x=b_{ \pm}(t)$. The substrate is vibrated at angle $\psi$ with acceleration $\gamma(t)$ with respect to its vertical, with $s(x)$ and $f(x)$ prescribing the substrate profile and the contact angle variations, respectively. The hatching below the substrate profile is used to show the variations of the contact angle. It is shaded in grey between the maximum (white) and minimum (dark grey/black) values of $f(x)$ corresponding to the portion of the substrate shown. The acceleration due to gravity, $g$, is vertically downwards.

discussed in $\S 4.4$ ), we utilise the long-wave approximation to obtain an evolution equation for the droplet thickness, $H\left(X, T_{*}\right)$,

$$
\partial_{T_{*}} H+\partial_{X}(H \bar{U})=0 .
$$

Here, $\bar{U}$ is the height-averaged velocity of the fluid in the $X$ direction, given by

$$
\begin{aligned}
\bar{U}= & \frac{H(H+3 \Lambda)}{3 \mu}\left[\sigma \partial_{X}^{3}(H+S)-\rho(g \cos \phi+\Gamma \cos \psi) \partial_{X}(H+S)\right. \\
& +\rho(g \sin \phi+\Gamma \sin \psi)],
\end{aligned}
$$

where $g$ is the gravitational acceleration, with $\sigma$ and $\mu$ being the surface tension and dynamic viscosity of the fluid, respectively. Following SK, (2.2) is made nondimensional by introducing the variables

$$
x=\frac{X}{L}, \quad h=\frac{H}{L \tan \alpha_{s}}, \quad s=\frac{S}{L \tan \alpha_{s}}, \quad t=\frac{T_{*}}{\tau}, \quad \lambda=\frac{3 \Lambda}{L \tan \alpha_{s}}, \quad f=\frac{F}{\tan \alpha_{s}}, \quad(2.4 a-f)
$$

where $\alpha_{s}$ is taken to be the average contact angle of the substrate, $\tau=3 \mu L /\left(\sigma \tan ^{3} \alpha_{s}\right)$ is the characteristic time scale of the problem and $L$ is its corresponding length scale, defined in terms of the cross-sectional area of the droplet, $A$, as $L=\sqrt{A \cot \alpha_{s}}$. With this choice of scales, (2.2) becomes

$$
\partial_{t} h+\partial_{x}\left\{h^{2}(h+\lambda)\left[\partial_{x}^{3}(h+s)-w_{1} \partial_{x}(h+s)+w_{2}\right]\right\}=0,
$$

which depends on $w_{1}(t)$ and $w_{2}(t)$, defined as

$$
w_{1}(t)=B o(\cos \phi+r \gamma(t) \cos \psi) \quad \text { and } \quad w_{2}(t)=B o(\sin \phi+r \gamma(t) \sin \psi) \cot \alpha_{s}, \quad(2.6 a, b)
$$


where $B o=\rho g L^{2} / \sigma$ is the Bond number, $r=\Gamma_{0} / g$ characterises the vibration strength by comparing the gravitational acceleration with the amplitude of the acceleration of the substrate vibrations, $\Gamma_{0}$, and $\gamma(t)=\Gamma(t \tau) / \Gamma_{0}$ is the dimensionless substrate acceleration.

We solve (2.5) subject to boundary conditions imposed at the right and left boundary points, $x=b_{ \pm}(t)$ (see figure 1 ),

$$
\begin{aligned}
h & =0, \\
\partial_{x} h & =\mp \alpha_{ \pm}, \\
\dot{b}_{ \pm} & =\lambda h \partial_{x}^{3} h,
\end{aligned}
$$

where the dot denotes differentiation with respect to $t$ and $\alpha_{ \pm}$are given by

$$
\alpha_{ \pm}=f_{ \pm} \frac{1+s_{ \pm}^{\prime 2} \tan ^{2} \alpha_{s}}{1 \pm s_{ \pm}^{\prime} f_{ \pm} \tan ^{2} \alpha_{s}}
$$

with $s_{ \pm}^{\prime}=\left.\partial_{x} s\right|_{x=b_{ \pm}}$and $f_{ \pm}=f\left(b_{ \pm}\right)$.

Conditions $(2.7 a)$ require that the droplet thickness vanishes at the two contact points and $(2.7 b)$ ensures that the droplet free surface meets the topography at an angle prescribed locally through the heterogeneity function $f(x)$. It is found by a simple geometric argument, namely by considering the slope of the free surface in dimensional units at the contact points and requiring that the tangents at the free surface and the substrate form an angle $\Theta(X)$, satisfying $\tan (\Theta(X))=F(X)$. Hence, at the right and left contact points, the slopes are given by $\partial_{X} H=\mp(\tan (\Theta \mp \Psi) \pm \tan \Psi)$, respectively, where $\tan \Psi=\partial_{X} S$. We deduce $(2.7 b)$ by expressing these conditions in dimensionless variables (Savva \& Kalliadasis 2009).

The third set of conditions, $(2.7 c)$, are of kinematic type and are readily found from (2.5) and the asymptotics of the solution as the contact points are approached. Thus, the problem is closed without the need to impose any semi-empirical, Cox-Voinovtype relations to advance the contact line, as done, for example, in the works of Benilov (2010, 2011) and Benilov \& Billingham (2011). Lastly, mass is conserved at all times, and we take

$$
\int_{b_{-}}^{b_{+}} h(x, t) \mathrm{d} x=1,
$$

with our chosen dimensionalisation.

With these scalings, $\dot{b}_{ \pm}$correspond to $3 C a_{ \pm} / \tan ^{3} \alpha_{s}$, where $C a_{ \pm}=\mu V_{ \pm} / \sigma$ can be viewed as local capillary numbers for the (dimensional) velocities $V_{ \pm}$of the right and left contact points, respectively. In the experiments by Brunet et al. (2007, 2009), capillary numbers of $O\left(10^{-3}\right)$ were reported, consistent with the main assumption of our theory which follows in the next section, namely that $\left|\dot{b}_{ \pm}\right| \ll 1$. More specifically, in the next section we will see that analytical progress is possible for small droplets with $B o \ll 1$, for substrate vibrations with $r=O(1)$ and in the limit where the time scale of oscillations is much larger than the viscous time scale, $\tau$. This underlying assumption is not particularly restrictive, especially given that, for the type of liquids used in the experiments of Brunet et al. (2007, 2009), $\tau$ is found to be of the order of milliseconds. It is to be noted, however, that a fair comparison with the aforementioned experiments is not possible because of both the 2D geometry considered here and the large contact angles that Brunet et al. have measured in their experiments. We will also explore a broader range of parameter values that do not conform with the assumptions of the theory by considering directly the governing PDE (2.5) and the conditions (2.7). 


\section{Matched asymptotics}

The analysis we follow is nearly identical to that in SK, the main difference being two additional terms arising from $\dot{w}_{1}$ and $\dot{w}_{2}$ that appear in the IDEs for $b_{ \pm}(t)$. Even though these terms are small in general, their inclusion typically improves the agreement between the numerical solution to (2.5) and the solution to the IDEs obtained from matching. As in SK, we assume quasi-static dynamics, and, by considering the flow in the bulk of the droplet and in the two regions near the two contact points separately, we match asymptotically the solutions within the two overlap regions that connect the contact point regions with the droplet bulk. The aim of doing so is to obtain a system of IDEs for $b_{ \pm}(t)$.

\subsection{Analysis}

The outer-region dynamics is examined by mapping the time-dependent domain over which (2.5) is solved, $b_{-}(t) \leqslant x \leqslant b_{+}(t)$, to the fixed interval $-1 \leqslant y \leqslant 1$ using

$$
x=d(t) y+\ell(t)
$$

with

$$
d=\frac{b_{+}-b_{-}}{2} \quad \text { and } \quad \ell=\frac{b_{+}+b_{-}}{2}
$$

defined as the droplet radius and midpoint, respectively.

By neglecting slip effects in the outer region and using (3.1), equation (2.5) transforms to

$$
\partial_{t} h-\frac{\dot{b}_{+}(1+y)+\dot{b}_{-}(1-y)}{2 d} \partial_{y} h+\frac{1}{d^{4}} \partial_{y}\left\{h^{3}\left[\partial_{y}^{3}(h+s)-d^{2} w_{1} \partial_{y}(h+s)+d^{3} w_{2}\right]\right\}=0 .
$$

Since the outer-region dynamics cannot capture the behaviour in the inner regions, (3.3) is solved with (2.7a) and (2.9). From the discussion in the previous section, we expect $\left|\dot{b}_{ \pm}(t)\right| \ll 1$ and, by assuming low-frequency vibrations so that $|\dot{\gamma}(t)| \ll 1$, we introduce a quasi-static expansion of the form

$$
h=h_{0}\left(y ; b_{ \pm}(t), \gamma(t)\right)+h_{1}\left(y ; b_{ \pm}(t), \gamma(t), \dot{b}_{ \pm}(t), \dot{\gamma}(t)\right)+\cdots,
$$

with $h_{0} \gg h_{1}$ and time entering the problem through $\gamma$ and $b_{ \pm}$and their time derivatives. Using (3.4) in (3.3), we find that the leading-order outer solution, $h_{0}$, satisfies

$$
\partial_{y}^{3}\left(h_{0}+s\right)-d^{2} w_{1} \partial_{y}\left(h_{0}+s\right)+d^{3} w_{2}=0
$$

with $h_{0}\left(b_{ \pm}\right)=0$ and $\int_{b_{-}}^{b_{+}} h_{0} \mathrm{~d} y=2 / d$. Equation (3.5) is essentially the derivative of the Young-Laplace equation as expressed in long-wave form, a simplified version of which was considered by Benilov (2010) in the absence of topography. The solution is identical to equation (3.5) in SK, namely

$$
\begin{aligned}
h_{0}(y)= & \frac{\frac{1}{2 d}+\bar{s}-\frac{1}{2}\left(s_{+}+s_{-}\right)}{\cosh k-k^{-1} \sinh k}(\cosh k-\cosh k y)+\frac{w_{2} d^{3}}{k^{2}}\left(y-\frac{\sinh k y}{\sinh k}\right) \\
& +\frac{1}{2}\left(s_{+}+s_{-}\right)+\frac{1}{2}\left(s_{+}-s_{-}\right) \frac{\sinh k y}{\sinh k}-s(\mathrm{~d} y+\ell),
\end{aligned}
$$


with $k=d \sqrt{w_{1}}, \bar{s}=\frac{1}{2} d^{-1} \int_{b_{-}}^{b_{+}} s(x) \mathrm{d} x$ and $s_{ \pm}=s\left(b_{ \pm}\right)$. Equation (3.6) gives the thickness of the droplet along the substrate at leading order, so that $h_{0}+s$ prescribes the shape of the free surface. Note that the validity of this analysis is limited to the cases where $w_{2} \ll 1$, since (3.6) can become negative somewhere for sufficiently large values of $w_{2}$, which is clearly non-physical (see also the discussion in Savva \& Kalliadasis (2013)).

The higher-order correction, $h_{1}$, is assumed to depend linearly on $\dot{b}_{ \pm}$and $\dot{\gamma}$, so that, by taking

$$
\partial_{t} h \approx \dot{b}_{+} \partial_{b_{+}} h_{0}+\dot{b}_{-} \partial_{b_{-}} h_{0}+\dot{w}_{1} \partial_{w_{1}} h_{0}+\dot{w}_{2} \partial_{w_{2}} h_{0}+\cdots,
$$

and after some algebra, we obtain a linear differential equation for $h_{1}$,

$$
\partial_{y}^{3} h_{1}-d^{2} w_{1} \partial_{y} h_{1}=-\frac{d^{4} D(y)}{h_{0}^{3}},
$$

where

$$
D(y)=q_{+}(y) \theta_{+} \dot{b}_{+}+q_{-}(y) \theta_{-} \dot{b}_{-}+A_{1} \dot{w}_{1}+A_{2} \dot{w}_{2},
$$

with

$$
\begin{aligned}
q_{ \pm}(y)= & \frac{1}{2}\left(\frac{\cosh k y-\cosh k}{k \sinh k} \pm \frac{\sinh k y-y \sinh k}{k \cosh k-\sinh k}\right) \\
\theta_{ \pm}= & \frac{k^{2}}{d} \frac{\frac{1}{2 d}+\bar{s}-\frac{s_{+}+s_{-}}{2}}{k \operatorname{coth} k-1} \pm \frac{w_{2} d^{2}}{k^{2}}(k \operatorname{coth} k-1) \mp k \frac{s_{+}-s_{-}}{2 d} \operatorname{coth} k \pm s_{ \pm}^{\prime} \\
A_{1}= & -\frac{d^{2}}{4 k^{4}}(k \operatorname{coth} k-1)\left[\left(s_{+}-s_{-}\right) k^{2}-2 d^{3} w_{2}\right]\left[q_{-}(1+y)+(1-y) q_{+}\right] \\
& -\frac{d^{2}}{4 k^{4}}\left[\left(s_{+}-s_{-}\right) k^{2}-4 d^{3} w_{2}\right]\left(1-y^{2}+2 q_{+}+2 q_{-}\right) \\
& -\frac{d^{3}}{4 k^{2}}\left(\theta_{+}+\theta_{-}+s_{+}^{\prime}+s_{-}^{\prime}\right)\left[q_{+}(y-1)+q_{-}(y+1)\right] \\
A_{2}= & -\frac{d^{3}}{k^{2}}\left(\frac{1-y^{2}}{2}+q_{+}+q_{-}\right) .
\end{aligned}
$$

Hence, two additional terms arise compared to the non-vibrating case in SK, $A_{1}$ and $A_{2}$. Equation (3.8) is to be solved subject to homogeneous boundary conditions, namely

$$
h_{1}( \pm 1)=0 \text { and } \quad \int_{-1}^{+1} h_{1}(y) \mathrm{d} y=0 .
$$

To perform the matching, we are only interested in the asymptotic behaviour of $h_{1}$ as $y \rightarrow \pm 1$. Following SK, in which the procedure to obtain the asymptotics of equations like (3.8) is outlined, we find that

$$
\partial_{y} h_{1} \sim-\dot{b}_{ \pm} \frac{d}{\theta_{ \pm}^{2}}\left[\ln \frac{e}{2}(1 \mp y)+\beta_{ \pm}\right],
$$

where $\beta_{ \pm}$are functions of time given by

$$
\beta_{ \pm}=\int_{-1}^{+1}\left[\frac{1}{1 \mp y}-\frac{d^{3} \theta_{ \pm}^{2} q_{ \pm}(y)}{h_{0}^{3} \dot{b}_{ \pm}} D(y)\right] \mathrm{d} y
$$


obtained by multiplying (3.8) by $q_{ \pm}(y)$ and integrating with respect to $y$ from -1 to +1 , followed by a series of integrations by parts and application of the conditions (3.14). Therefore, the behaviour of $\partial_{x} h$ is given by

$$
\mp \partial_{x} h \sim \theta_{ \pm} \pm \frac{\dot{b}_{ \pm}}{\theta_{ \pm}^{2}}\left[\ln \left(\mp e \frac{x-b_{ \pm}}{2 d}\right)+\beta_{ \pm}\right], \quad \text { as } x \rightarrow b_{ \pm},
$$

where clearly $\theta_{ \pm}$can be identified as the apparent contact angles. For small-amplitude topographies, the apparent contact angle is simply given by $\theta_{ \pm}$but with $s \equiv 0$ (see also discussion in $\S 5.3$ ).

This outer-region behaviour needs to be matched asymptotically with the behaviours from the inner regions near the moving fronts, which account for the influence of slip. To probe into these regions, for which $x-b_{ \pm}=O(\lambda)$, we introduce the stretched variables

$$
\xi_{ \pm}=\mp\left(x-b_{ \pm}\right) \alpha_{ \pm} / \lambda \quad \text { and } \Phi_{ \pm}=h / \lambda
$$

to transform $(2.5)$ to

$$
\pm \dot{b}_{ \pm} \partial_{\xi_{ \pm}} \Phi_{ \pm}+\alpha_{ \pm}^{3} \partial_{\xi_{ \pm}}\left[\Phi_{ \pm}^{2}\left(\Phi_{ \pm}+1\right) \partial_{\xi_{ \pm}}^{3} \Phi_{ \pm}\right]=0 .
$$

In (3.19), we retained only the $O\left(\lambda^{0}\right)$ terms and neglected the effects of topography, gravity and substrate vibrations, since $\lambda^{2}\left|w_{1}\right|, \lambda^{2}\left|w_{2}\right|$ and $\lambda\left|\partial_{x}^{3} s\right|$ are all assumed to be small. The asymptotics of (3.19) have been treated in SK and elsewhere (see e.g. Hocking 1983; Savva \& Kalliadasis 2009, 2012; Vellingiri, Savva \& Kalliadasis 2011). Hence, using quasi-static dynamics and a wedge-shaped free surface, to leading order we find that

$$
\partial_{\xi_{ \pm}} \Phi_{ \pm} \sim 1 \mp \frac{\dot{b}_{ \pm}}{\alpha_{ \pm}^{3}}\left(\ln \xi_{ \pm}+1\right), \quad \text { as } \xi_{ \pm} \rightarrow+\infty .
$$

A transformation back to the original variables gives

$$
\mp \partial_{x} h \sim \alpha_{ \pm} \pm \frac{\dot{b}_{ \pm}}{\alpha_{ \pm}^{2}}\left[\ln \left(\mp \alpha_{ \pm} \frac{x-b_{ \pm}}{\lambda}\right)+1\right] \quad \text { as } \mp \frac{x-b_{ \pm}}{\lambda} \rightarrow \infty
$$

for the asymptotic behaviours of the inner regions, from which, when raised to the third power, one obtains the equivalent Cox-Voinov law, with (dimensionless) microscopic length $\lambda /\left(\alpha_{ \pm} \mathrm{e}\right)$ (see e.g. Bonn et al. 2009, and references therein). The behaviours in (3.21) are to be matched with those from the outer region, (3.17). As it turns out (see e.g. Savva \& Kalliadasis 2009), it is possible to match the third power of (3.17) and (3.21) and we find

$$
\pm \dot{b}_{ \pm}\left[\ln \frac{2 d \alpha_{ \pm}}{\lambda}-\beta_{ \pm}\right]=\theta_{ \pm}^{3}-\alpha_{ \pm}^{3} .
$$

Equations (3.22) are reminiscent of Cox-Voinov-type imposed boundary conditions in studies with moving contact lines (see e.g. Hocking 1992; Wilson, Hunt \& Duffy 2000 ), in which the velocity of a moving contact line, $V$, is prescribed by a semiempirical relation of the form $V=\kappa\left(\theta_{\text {macro }}^{3}-\theta_{\text {micro }}^{3}\right)$, where $\theta_{\text {macro }}$ is the macroscopic apparent contact angle, $\theta_{\text {micro }}$ is the microscopic angle at the contact line and $\kappa$ is some constant of proportionality. Thus, instead of assuming a relation of this form, we derive it as a product of the matching procedure, which allows us to determine 
$\kappa$ as a function of the contact line fronts and substrate heterogeneities. The matching conditions, (3.22), may be solved for $\dot{b}_{ \pm}$to yield

$$
\dot{b}_{ \pm}= \pm \frac{\delta_{ \pm} G_{\mp}+\delta_{\mp} \theta_{ \pm} G_{0}}{G_{-} G_{+}-\theta_{-} \theta_{+} G_{0}^{2}}
$$

with

$$
\begin{aligned}
G_{ \pm} & =\ln \frac{2 d \alpha_{ \pm}}{\lambda}-\int_{-1}^{+1}\left[\frac{1}{1 \mp y}-\frac{d^{3} \theta_{ \pm}^{3} q_{ \pm}^{2}}{h_{0}^{3}}\right] \mathrm{d} y, \\
G_{0} & =\theta_{+} \theta_{-} d^{3} \int_{-1}^{+1} \frac{q_{+} q_{-}}{h_{0}^{3}} \mathrm{~d} y, \\
\delta_{ \pm} & =\frac{1}{3}\left(\theta_{ \pm}^{3}-\alpha_{ \pm}^{3}\right) \mp d^{3} \theta_{ \pm}^{2} \int_{-1}^{+1} \frac{q_{ \pm}\left(A_{1} \dot{w}_{1}+A_{2} \dot{w}_{2}\right)}{h_{0}^{3}} \mathrm{~d} y .
\end{aligned}
$$

Thus, equations (3.23) constitute a coupled system of IDEs, because determining $\dot{b}_{ \pm}$ requires the evaluation of the integrals, in $G_{ \pm}, G_{0}$ and $\delta_{ \pm}$. It is important to emphasise that (3.23) are nearly the same as those obtained in SK, the only difference being in the expressions for $\delta_{ \pm},(3.26)$, where we now include the terms containing $\dot{w}_{1}$ and $\dot{w}_{2}$. As previously mentioned, the inclusion of these terms does not influence the dynamics significantly, since they merely introduce $O\left(T^{-1}\right)$ corrections, which are assumed to be small. However, they sometimes tend to improve the agreement with the solutions to the governing PDE, (2.5), particularly at times when the moving fronts slow down. With this analysis, we were able to map the slow dynamics of the infinitedimensional dynamical system corresponding to the governing PDE, (2.5), to a 2D non-autonomous system of IDEs. In the following sections, we will be examining this set of equations under different conditions. Even though the substrate vibrations can be prescribed by any differentiable function $\gamma(t)$, we will be focusing our discussion on simple harmonic vibrations, so that the substrate experiences acceleration

$$
\gamma(t)=\cos \frac{2 \pi t}{T},
$$

where $T$ is an appropriately non-dimensional period of oscillation.

\subsection{Numerical methods; verification of asymptotics}

The validity of the matching procedure can be readily verified by performing sets of different computations to compare the solutions to the PDE (2.5) subject to (2.7) and the equations obtained by matching, (3.23). As in SK, the governing PDE is solved using a pseudospectral discretisation for the spatial coordinate (similar to the method employed in Savva \& Kalliadasis (2009)) and time marching is performed using the method of lines and the ode15s routine in MATLAB ${ }^{\circledR}$. The IDEs are solved using the Legendre-Gauss quadrature to obtain the associated integrals and a RungeKutta scheme for time stepping. Whilst there can exist countless ways of choosing the heterogeneity profiles, the focus of the present study will be limited primarily to single-wavelength topographical heterogeneities, since the phenomenology of the problem is not expected to change qualitatively. However, a few examples will be considered with chemical heterogeneities as well. 

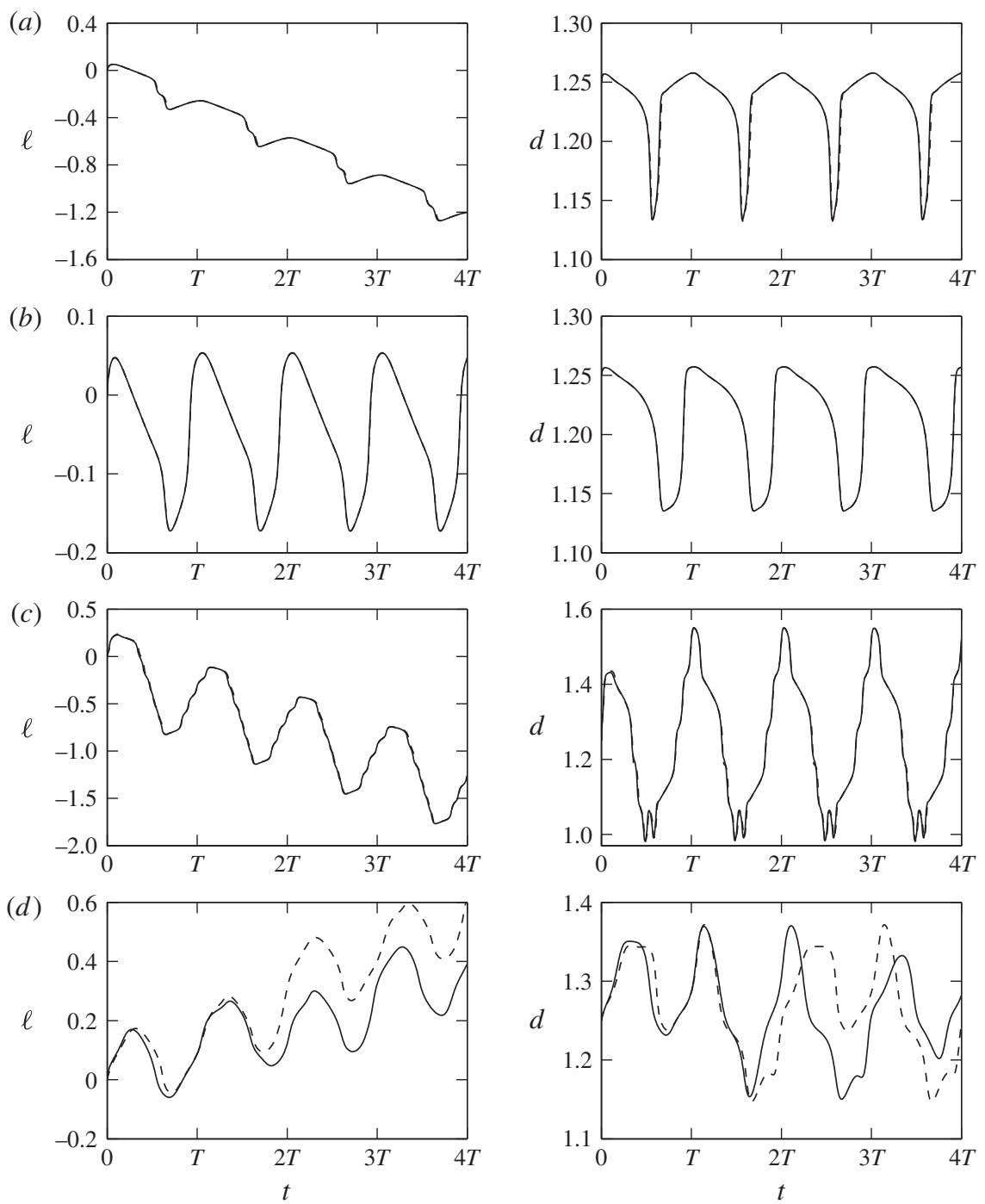

FIGURE 2. Plots of the evolution of $\ell(t)$ and $d(t)$ for a heterogeneous substrate with $s(x)=0.01 \cos 20 x$ and $f(x)=1$, comparing sample solutions to the governing PDE (solid curves), (2.5), and the IDEs obtained by matching, (3.23), (dashed curves). (a) The parameters for the computation are $T=100, \lambda=0.005, B o=0.1, r=6, \alpha_{s}=15^{\circ}$, $\phi=2^{\circ}, \psi=10^{\circ}$ and $b_{ \pm}(0)= \pm 1.25$. (b) As in panel $(a)$, but with $\lambda=10^{-4}$. (c) As in panel $(a)$, but with $r=40$ and $\psi=2^{\circ}$. (d) As in panel $(a)$, but with $B o=0.2$ and $T=20$. Note the excellent agreement between the IDEs and the governing PDE in panels $(a-c)$, where the dashed and solid curves are nearly indistinguishable. In panel $(d)$ the agreement is poor, which suggests that for these parameters the quasi-static assumption is no longer applicable.

By plotting the evolution of the droplet radius, $d(t)$, and midpoint, $\ell(t)$, we show in figure 2 a small selection of different possible behaviours that can be observed for a droplet moving on a topographically heterogeneous substrate whose profile is prescribed by $s(x)=0.01 \cos 20 x$ and is inclined at angle $\phi=2^{\circ}$. In figure 2(a) 
we show that the choice of parameters mentioned in the figure caption yields a pronounced climbing behaviour and relaxation-type oscillations in the droplet width. In figure $2(b)$ we see that changing the slip length yields a markedly different behaviour compared to figure 2(a), with the droplet eventually being trapped in the heterogeneity features with no net uphill or downhill motion. This is contrary to our expectation that the dynamics depend weakly on the slip length, given that $\lambda$ appears in the logarithm of (3.23). Even though, indeed, the dynamics depend weakly on $\lambda$, it is also possible that changes in slip, which may cause minute changes in speed, can be crucial when the contact line tries to overcome a topographical feature. Note that in the present study we fixed $\lambda=0.005$ in most of our computations, despite the fact that our numerical scheme can accurately capture the dynamics with slip lengths that are at least two orders of magnitude smaller than this value. A larger slip length was chosen because smaller slip lengths make the PDE stiffer to solve numerically, which can be inefficient especially for tracing full regime diagrams. Besides, the interesting behaviours are observed at small Bo, which would correspond to smaller droplets and the non-dimensional $\lambda$, which scales with the size of the droplet, will tend to be larger. Lastly, we do not expect the results to change qualitatively had we chosen a smaller $\lambda$.

If we alter the vibration strength in figure $2(a)$, we observe in figure $2(c)$ that the droplet moves on average slightly further up the inclined plane, noting also the higheramplitude oscillations in $\ell(t)$ compared to figure 2(a). As a result, during the course of a vibration period, the droplet travels across more peaks and valleys of the substrate topography, and this is manifested in the secondary oscillations in the evolution of $d(t)$.

It should be noted that in figure $2(a-c)$ we plotted the solution to both the IDEs, (3.23), and the governing PDE, (2.5), and we observe that they are indistinguishable. This is typically the case when Bo, $\psi, \phi$ and $r$ are relatively small and when $T$ is large, although it is impossible to pinpoint precise regimes of validity of our theory. For example, in figure $2(d)$, we show a calculation where our theory fails to capture the behaviour of the PDE. More importantly, the motion appears to be aperiodic compared to the other cases presented in figure 2, which exhibit periodic behaviour after some initial transient. This effect is frequently observed for substrates with small amplitude and wavelength, but it is difficult to predict when it might occur. This behaviour is presumably a result of some type of bifurcation at which the system switches to a new periodic behaviour having a period equal to a multiple of the period of forcing, $T$. Although this effect is rather interesting, we will not elaborate on it any further, apart from presenting some additional examples later on, in $§ 5.2$.

\section{Ideal substrates}

Before looking into the effects of substrate heterogeneities, we briefly revisit the ideal-substrate case $(s(x)=0, f(x)=1)$, first investigated numerically with a precursor film by John \& Thiele (2010). The motivation behind this discussion is to use the preceding analysis to extract analytically a climbing criterion valid for weak vibrational and gravitational effects, to contrast the present analysis with related works (Benilov 2010, 2011; Benilov \& Billingham 2011) and to study briefly how the dynamics is affected when weak inertial effects are also included.

\subsection{Mean drift velocity}

When we are dealing with an ideal substrate, it is easy to see that $\dot{d}=\left(\dot{b}_{+}-\dot{b}_{-}\right) / 2$ and $\dot{\ell}=\left(\dot{b}_{+}+\dot{b}_{-}\right) / 2$ both depend on $t$ and $d(t)$, but not on $\ell(t)$. This is to be expected, 
since the dynamics on an ideal substrate is characterised by translational invariance. Hence, one can solve for the evolution of the droplet radius independently from its displacement along the substrate. In fact, when $w_{1}(t)$ and $w_{2}(t)$ are both small, we can obtain an approximate expression for the drift velocity, $\dot{\ell}(t)$. By neglecting $O\left(\dot{w}_{1}\right)$ and $O\left(\dot{w}_{2}\right)$ terms, which corresponds to the low-frequency limit, $T \rightarrow \infty$, we obtain the following expansion for $d(t)$,

$$
d(t)=\frac{\sqrt{6}}{2}\left(1+\frac{1}{20} w_{1}+\frac{9}{5600} w_{1}^{2}+\frac{\ln \frac{6}{\lambda^{2}}-8}{8 \ln \frac{6}{\lambda^{2}}} w_{2}^{2}\right)+O\left(w_{1}^{3}, w_{1} w_{2}^{2}\right),
$$

found by taking $\dot{d}=O\left(\dot{w}_{1}, \dot{w}_{2}\right)$ and matching powers of $w_{1}$ and $w_{2}$. Using this expansion in $\dot{\ell}$, we find after some algebra

$$
\dot{\ell}(t)=\frac{w_{2}}{\ln \frac{6}{\lambda^{2}}}-\frac{3 w_{1} w_{2}}{5 \ln ^{2} \frac{6}{\lambda^{2}}}+O\left(w_{2}^{3}, w_{1}^{2} w_{2}\right)
$$

From (4.2) we can obtain the mean drift velocity, defined as

$$
\bar{v}=\frac{1}{T} \int_{0}^{T} \dot{\ell}(t) \mathrm{d} t,
$$

and hence, since by assumption $B o \ll 1$, we have

$$
\bar{v}=\frac{B o}{\tan \alpha_{s} \ln \frac{6}{\lambda^{2}}}\left(\sin \phi-\frac{3 \sin 2 \psi}{20 \ln \frac{6}{\lambda^{2}}} r^{2} B o-\frac{3 \sin 2 \phi}{10 \ln \frac{6}{\lambda^{2}}} B o\right)+O\left(B o^{3}, B o^{3} r^{2}\right) .
$$

It is apparent from this analysis that the non-zero time-averaged velocities of the contact points arise due to the nonlinear term $w_{1} w_{2}$ in (4.2), whereas the linear term averages to net downhill motion. It is also important to note that, unlike other analyses with inclined 2D droplets (see e.g. Benilov 2010, 2011; Benilov \& Billingham 2011), (4.4) can predict that uphill motion can be observed with low-frequency harmonic vibrations, which is consistent qualitatively with the numerical work of John \& Thiele (2010) with a precursor film model. Uphill motion at low frequencies was also found by Benilov (2011) and Benilov \& Cummins (2013) in their analysis of the 3D problem.

Numerical solutions of the governing equation (2.5) suggest that the ideal-substrate case settles, after some brief initial transient due to spreading, to a motion that exhibits periodically evolving speeds and free-surface shapes for the harmonic forcing prescribed through $\gamma(t)$, (3.27). Hence, to determine $\bar{v}$ numerically as a means to verify (4.4), we have neglected the transient dynamics and focused on extracting the long-time behaviour. This has been achieved by treating time in (2.5) as a periodic variable, which transforms the problem into solving a 'steady' PDE in two dimensions. Solutions to this problem were obtained efficiently and accurately by discretising the governing PDE spectrally in both time and space and utilising Newton iterations until convergence was achieved. 
(a)

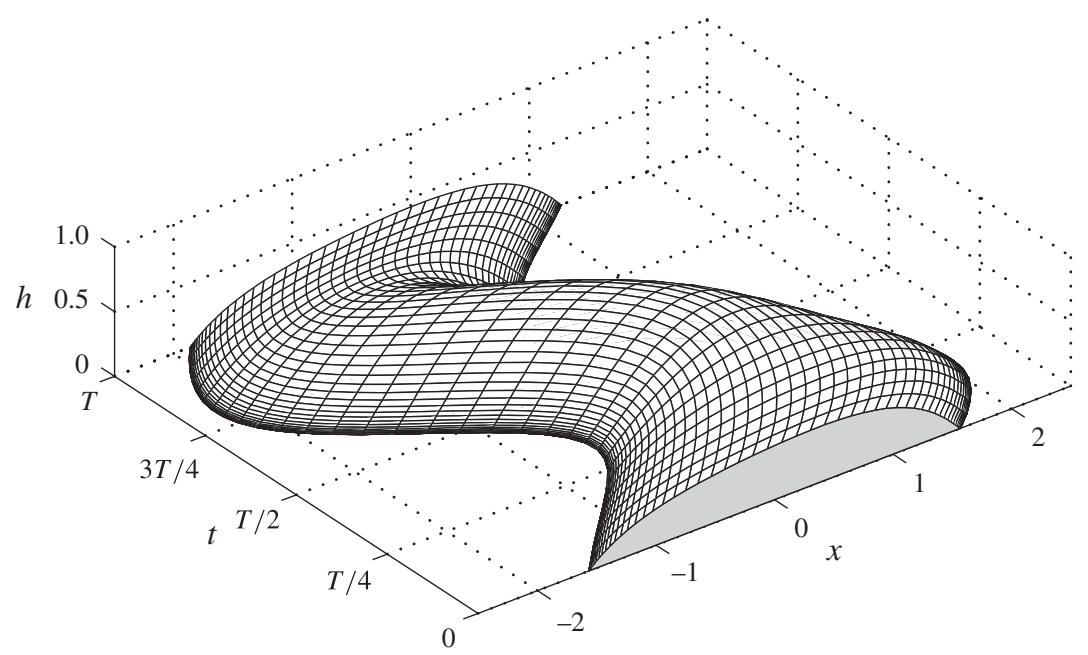

(b)

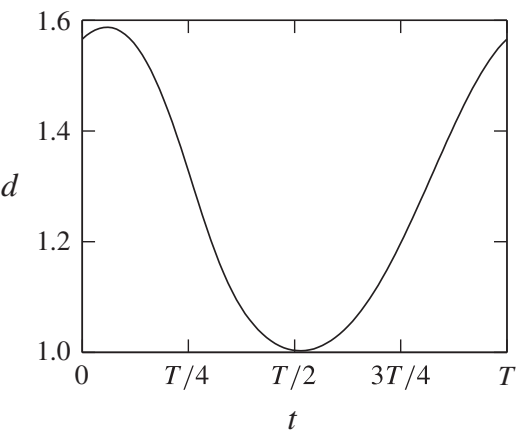

(c)

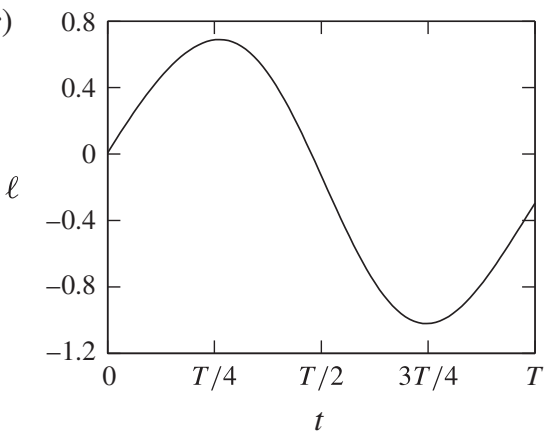

FIgURE 3. Long-time behaviour of a droplet with $B o=0.2$ that moves on an ideal substrate with $\lambda=0.005, \alpha_{s}=15^{\circ}$ and $\phi=2^{\circ}$, vibrated at angle $\psi=2^{\circ}$, period $T=100$ and $r=24$. (a) Surface plot of $h(x, t)$ showing the evolution of the droplet free surface during a vibration period. (b) Periodic evolution of $d(t)$. (c) Plot of $\ell(t)$ with $\ell(0)=0$, which demonstrates the uphill translation of the droplet at the end of each period.

Figure 3 shows the result of such a computation for a droplet with $B o=0.2$ moving on a substrate with $\lambda=0.005, \alpha_{s}=15^{\circ}$ and $\phi=2^{\circ}$, which is vibrated at angle $\psi=2^{\circ}$, period $T=100$ and $r=24$. The particular configuration yields $\bar{v}=-0.0030$, i.e. uphill motion is observed (the linear theory, (4.4), predicts $\bar{v}=-0.0038$; this discrepancy is expected since the chosen parameters lie beyond the regime where (4.4) is an acceptable approximation). A plot of $h(x, t)$ is given in figure $3(a)$, which shows the free-surface oscillations during the course of a period. Figure $3(b)$ shows the periodically evolving width of the droplet, $d(t)$, over a period, and figure $3(c)$ shows the plot of $\ell(t)$ with $\ell(0)=0$ to demonstrate the uphill shift of the droplet midpoint when $t=T$, where we should reiterate that $\dot{\ell}(t)$ is periodic with non-zero mean and gives $\ell(t)$ as a superposition of harmonic oscillations and a constant-velocity translation. 


\subsection{Horizontal substrates}

When the substrate is horizontal $(\phi=0)$, the sign of the mean drift velocity is determined solely by the sign of $\psi$, as expected from (4.4), which becomes

$$
\bar{v}=-\frac{3 r^{2} B o^{2} \sin 2 \psi}{20 \tan \alpha_{s} \ln ^{2} \frac{6}{\lambda^{2}}}+O\left(B o^{3}, B o^{3} r^{2}\right) .
$$

From (4.5) we anticipate that $|\bar{v}|$ is maximised when $\psi= \pm 45^{\circ}$. A heuristic argument why this is so can be made by considering the extreme values of $\psi, 0$ and $90^{\circ}$, where, due to symmetry, we do not expect any net motion. At $\psi=0^{\circ}$ the contact points evolve symmetrically since the substrate is vibrated in the direction normal to the substrate. At $\psi=90^{\circ}$ the substrate is shaken horizontally, and the motion in each half-cycle is reversed in the other half-cycle. In these extreme values, $\bar{v}=0$. Hence, it is natural to expect $\bar{v}$ for the linearised problem to attain its maximum value when $\psi$ is close to $45^{\circ}$.

In figure 4 we show a number of low-frequency calculations that demonstrate that, when we have both $B o, r B o \ll 1$, we get very good agreement between theory and numerical experiments, with the maximum $|\bar{v}|$ indeed occurring around $\psi=45^{\circ}$. Interestingly, when the assumptions of our linear theory no longer hold, the calculations tend to exhibit excellent agreement with (4.5) only for small $\psi$, with the maximum speed occurring at progressively smaller vibration angles as $B o$ and $r$ both increase. A plausible explanation why the optimum angle is reduced can be given in terms of the aforementioned mechanism of uphill motion suggested by John \& Thiele (2010). Hence, since, according to this mechanism, the vertical component of vibration is ultimately responsible for drop mobility along the substrate, the reduction of the optimum angle is indicative of the need for stronger vibrations along the normal to the substrate (see curves $C$ and $D$ in figure 4), which may be attributed to the stronger coupling between the horizontal and vertical components of acceleration in the nonlinear regime.

\subsection{Inclined substrates}

\subsubsection{Climbing criterion}

The sign of $\bar{v}$ in (4.4) determines which way the droplet will move in the long-time limit. Hence, for an inclined substrate $(\phi>0)$, we get uphill motion when $\bar{v}<0$. Thus, the first two terms in the expansion of (4.4) reveal the two competing mechanisms that decide the direction of net transport. One is the component of gravity along the substrate, $B o \sin \phi$, and the other is the effect of substrate vibrations through $r^{2} B o^{2} \sin 2 \psi$, whose influence is determined by the vibration angle, which ultimately characterises the breaking of symmetry (see earlier discussion on figure 4). Moreover, since droplet transport is facilitated by larger values of $\lambda$, we also see that larger $\lambda$ promotes the uphill motion, albeit weakly, as it appears in the logarithm, and one would need to change $\lambda$ by orders of magnitude to see an appreciable effect. Hence, since $\phi, \quad \psi$ and $B o$ are assumed to be small, while $\ln \left(6 / \lambda^{2}\right)$ is typically of $O(10)$, uphill motion occurs if we choose $r \gg 1$, so that

$$
r^{2} \geqslant \frac{20 \sin \phi \ln \frac{6}{\lambda^{2}}}{3 B o \sin 2 \psi}+O\left(B o^{2}, B o^{3} r^{2}\right) .
$$




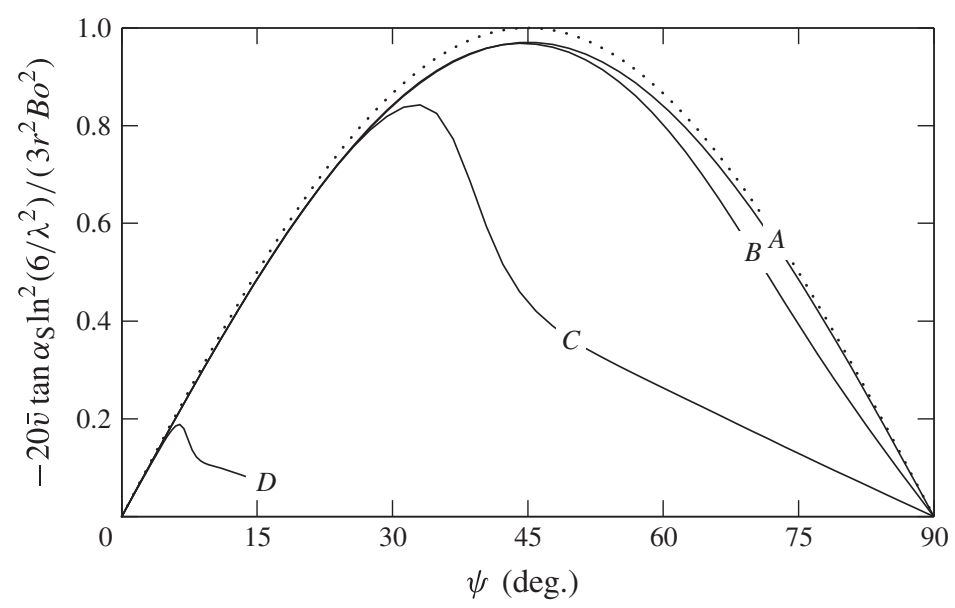

FIGURE 4. Influence of vibration angle on the mean drift velocity, $\bar{v}$, where we plot $-20 \bar{v} \tan \alpha_{s} \ln ^{2}\left(6 / \lambda^{2}\right) /\left(3 r^{2} B o^{2}\right)$ as a function of $\psi$ (in all computations, $T=1000, \lambda=$ $5 \times 10^{-3}$ and $\alpha_{s}=10^{\circ}$ ). The solid curves $A-C$ correspond to $B o=0.05$ with $r=1,5$ and 8 , respectively, and curve $D$ to $B o=1$ and $r=2$ (note that curve $D$ is computed up to $\psi=13^{\circ}$ because the problem becomes too stiff to be efficiently resolved). The dotted curve is the theoretical prediction from (4.5), $\sin 2 \psi$.

At the same time, we must require that $r B o \ll 1$ and $T \rightarrow \infty$ to be consistent with the derivation of (4.4). Note that this result agrees, at least qualitatively, with the numerical experiments of John \& Thiele (2010), who demonstrated that for small drops $r$ is inversely proportional to $\psi^{-1 / 2}$. Here we have shown rigorously how this relation arises naturally by linearising the equations obtained through asymptotic matching.

Figure 5 shows calculations that verify the climbing criterion, where we plot the square of the critical amplitude of the vibration acceleration, $r_{c}^{2} B o^{2}$, for which $\bar{v}=0$, as a function of $B o$. The agreement is typically excellent for very small values of $B o$, but it can be extended to higher $B o$ as both $\phi$ and $\psi$ are made smaller. Since $\psi$ is the only parameter that differs among the curves in figure 5 , we can also infer that using larger $\psi$ can generally promote the uphill droplet motion, since we need to vibrate the substrate less vigorously (i.e. lower $r_{c}$ ) to get the droplet to move against gravity. The same conclusion is reached by looking at (4.4), but one has to bear in mind the earlier discussion on horizontal substrates, since nonlinearities can shrink the range of vibration angles over which $\bar{v}$ increases monotonically with $\psi$.

\subsubsection{Regime diagram}

Figure 6 shows a typical regime diagram that illustrates the effect of the vibration strength, $r$, and period of oscillation, $T$, on the time-averaged motion of the droplet. As $T$ becomes larger, $\bar{v}$ tends to be independent of $T$, which is consistent with our derivation of the mean drift velocity, (4.4) (see also figure 7). In fact, the low-frequency critical vibration strength determined from the numerics is found to be $r_{c}=14.5$, which is less than $1.5 \%$ in error from the theoretically predicted value using (4.6), $r_{c}=14.3$. There also appears to be a cutoff frequency above which substrate vibrations, no matter how strong, cannot induce uphill motion. We will return to this point when we discuss weak inertial effects. This regime diagram is topologically 


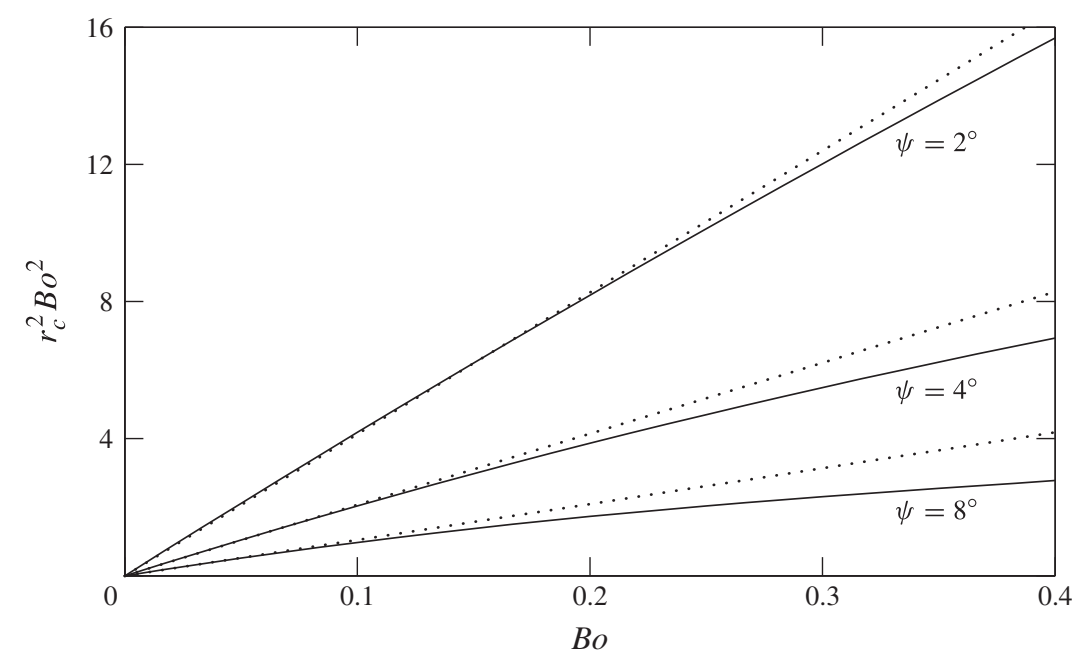

FIGURE 5. Plots of the critical amplitude of the vibration acceleration as a function of the Bond number to verify the climbing criterion, (4.6). The solid curves are the result of computations tracing $r_{c}$ so that $\bar{v}=0$ for different values of $\psi$ labelling each curve in the figure, plotted together with the corresponding prediction from theory (dotted lines). The other parameters used for the computations are $\lambda=5 \times 10^{-3}, \alpha_{s}=10^{\circ}$ and $\phi=2^{\circ}$.

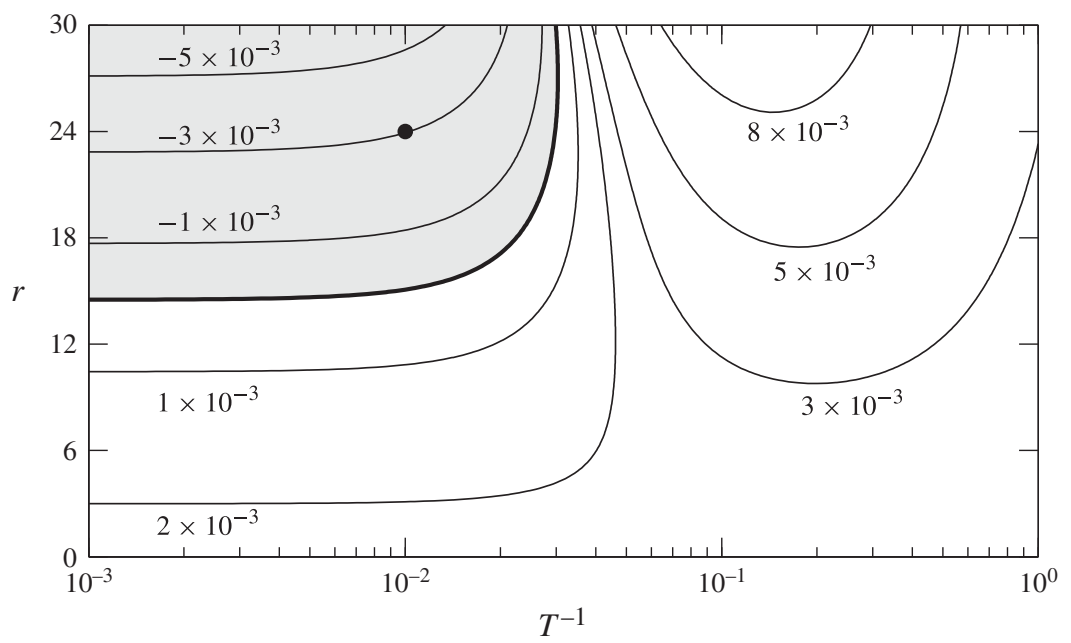

FIGURE 6. Regime diagram for varying $T$ and $r$ (see caption of figure 3 for the other parameters). The thin curves are contours of constant $\bar{v}$, whereas along the thick curve we have $\bar{v}=0$. The shaded region represents the regime of uphill motion; in the unshaded region the droplet would move downhill. The solid circle marks the parameters corresponding to the calculation in figure 3 .

similar to those obtained by Benilov \& Cummins (2013) for 3D droplets with large contact angles and by Thiele \& John (2010) for a 2D precursor film model, which is not surprising given the equivalence of slip and the precursor film models (Savva \& Kalliadasis 2011). 


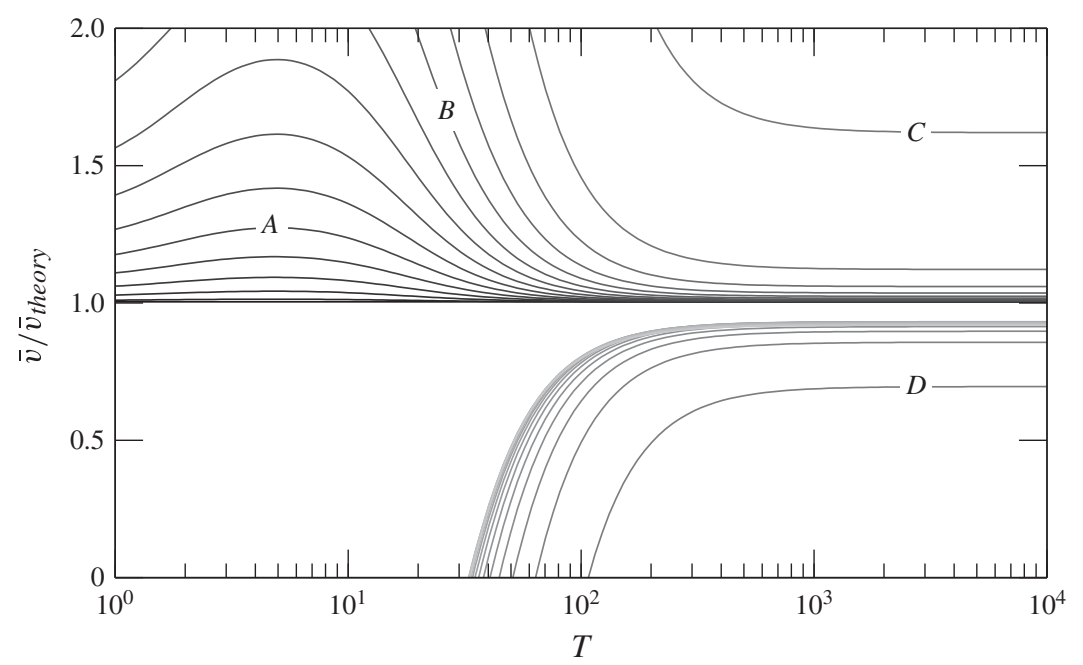

FIGURE 7. Plot of the ratio of the numerically determined $\bar{v}$ and the one predicted by (4.4) as a function of $T$ for $r=1,2, \ldots, 30$ (see caption of figure 3 for the remaining parameters). The curves are drawn with a lighter colour as $r$ is increased. Curves $A-D$ correspond to $r=5,10,14$ and 15 , respectively. As expected, $\bar{v}$ becomes independent of $T$ as $T \rightarrow \infty$. The largest difference between theory and numerics is observed when $r=14$ and $r=15$ (curves $C$ and $D$ ), since around $r=14.5$ we have $\bar{v}=0$ and round-off errors are naturally amplified when computing the ratio of the two vanishing speeds.

However, it is worth noting that the topology of the regime diagram differs in some respects from that observed in the experiments of Brunet et al. (2007, 2009). The most striking difference is that Brunet et al. observed that there can exist 'static' regions, where the droplet does not appear to exhibit measurable net motion, especially in the high-frequency regime. This behaviour cannot possibly be captured by the physical mechanisms assumed in the inertia-less, ideal-substrate and 2D model that we have utilised here, which only predicts either uphill or downhill motion. In the following sections, we will attempt to understand how the inclusion of weak inertia and substrate heterogeneities can possibly affect the droplet dynamics in an effort to identify the underlying mechanisms for the behaviour observed in experiments.

\subsection{Weak inertial effects}

In deriving (2.5) we have neglected the effects of fluid inertia, but it is also of interest to examine how its inclusion affects the regime diagram of figure 6 . We thus retain the inertial terms in the momentum equations, by adopting the so-called Kármán-Pohlhausen averaging method. Thus we consider the following appropriately non-dimensionalised system of PDEs:

$$
\begin{aligned}
O h\left\{\partial_{t} q+\partial_{x}\left[q^{2} \frac{\frac{6}{5} h^{2}+2 h \lambda+\lambda^{2}}{h(h+\lambda)^{2}}\right]\right\} & =h\left(\partial_{x}^{3} h-w_{1} \partial_{x} h+w_{2}\right)-\frac{q}{h(h+\lambda)}, \\
\partial_{t} h+\partial_{x} q & =0 .
\end{aligned}
$$


Here $q(x, t)$ is the flow rate in the $x$ direction and $O h$ is the Ohnesorge number,

$$
O h=\frac{\rho \sigma L \tan ^{5} \alpha_{s}}{9 \mu^{2}}=\frac{\text { inertia } \times(\text { surface tension })}{(\text { viscous forces })^{2}},
$$

which relates the viscous forces to inertial and surface tension forces and is assumed to be at most $O(1)$. The derivation of the long-wave equation is given in appendix A and is nearly identical to the derivation of the model utilised by López et al. (1997) to study the contact line instability of a thin film coating an inclined plane. Obviously, if we neglect inertia and take $O h=0$ in (4.7), we recover (2.5). To see the effect of inertia on the dynamics, we compare the regime diagram obtained for non-zero $O h$ with the one presented in figure 6 . To obtain $\bar{v}$ numerically, we adopt the same strategy, namely convert the moving-boundary problem to a fixed-boundary one and extract in the same manner the long-time periodic behaviour of the equations, namely by assuming periodicity in time of the flow rate, the velocities of the contact points and the evolution of the free surface of the droplet.

Figure $8(a)$ shows the regime diagram when $O h=5$, using the same parameters as in figure 6 . We observe that, with the inclusion of inertia, a secondary climbing regime appears at higher frequencies. Overall, we observe that inertia generally reduces $|\bar{v}|$, as expected. However, given that our model is valid for relatively small $\mathrm{Oh}$, it is unclear whether the pinned no-net-transport regimes observed in the experiments of Brunet et al. (2007, 2009) at high frequencies can be attributed to sufficiently strong inertial effects. Moreover, the pronounced free-surface deformations shown by Brunet et al. appear to be consistent qualitatively with the deformations that we observe in our model at higher frequencies and sufficiently strong vibrations.

In figure $8(b)$ we overlay the contours in figures 6 and $8(a)$ when $O h=0$ (solid curves) and $O h=5$ (dashed curves), respectively, together with those obtained for $O h=10$ (dotted curves). We observe that the secondary climbing regime when $O h=10$ is shifted towards lower frequencies compared to the $O h=5$ case and that speeds are further reduced. More importantly, the contours in the low-frequency region appear to be affected to a much lesser extent by inertia compared to the high-frequency region, which is most likely due to the fact that the substrate is less vigorously vibrated.

In an attempt to understand better the presence of the secondary climbing regime, we have computed regime diagrams for various values of Oh. An important outcome of this investigation is that the secondary climbing region is present even at $O h=0$, but it was not seen in figure 6, as it required $r$ to be of $O(100)$ and $T$ to be small. Hence, the inclusion of inertia shifts this climbing regime towards lower frequencies and smaller values of $r$ compared to the case when $O h=0$.

When $O h=0$ and $r=O(100)$, we observe more pronounced free-surface deformations, with the contact line fronts moving very little over a period of oscillation. As previously mentioned, a similar behaviour was reported in the experiments by Brunet et al. (2007), which is to be contrasted with the case of low-frequency oscillations, where we see less pronounced free-surface deformations but appreciable variation in the location of the moving fronts. In figure $9(a)$ we show the evolution of the free surface for an uphill-moving droplet at higher frequencies, plotting $h(x, t)$ over a vibration period at $T=1$ and $r=100(\bar{v} \approx$ $-0.0053)$, which is to be contrasted with the calculation shown in figure $3(a)$ with $T=100$ and $r=24(\bar{v} \approx-0.0030)$, keeping all the other parameters the same. Figure $9(b)$ shows the evolution of the droplet midpoint, $\ell(t)$, and the change in the 

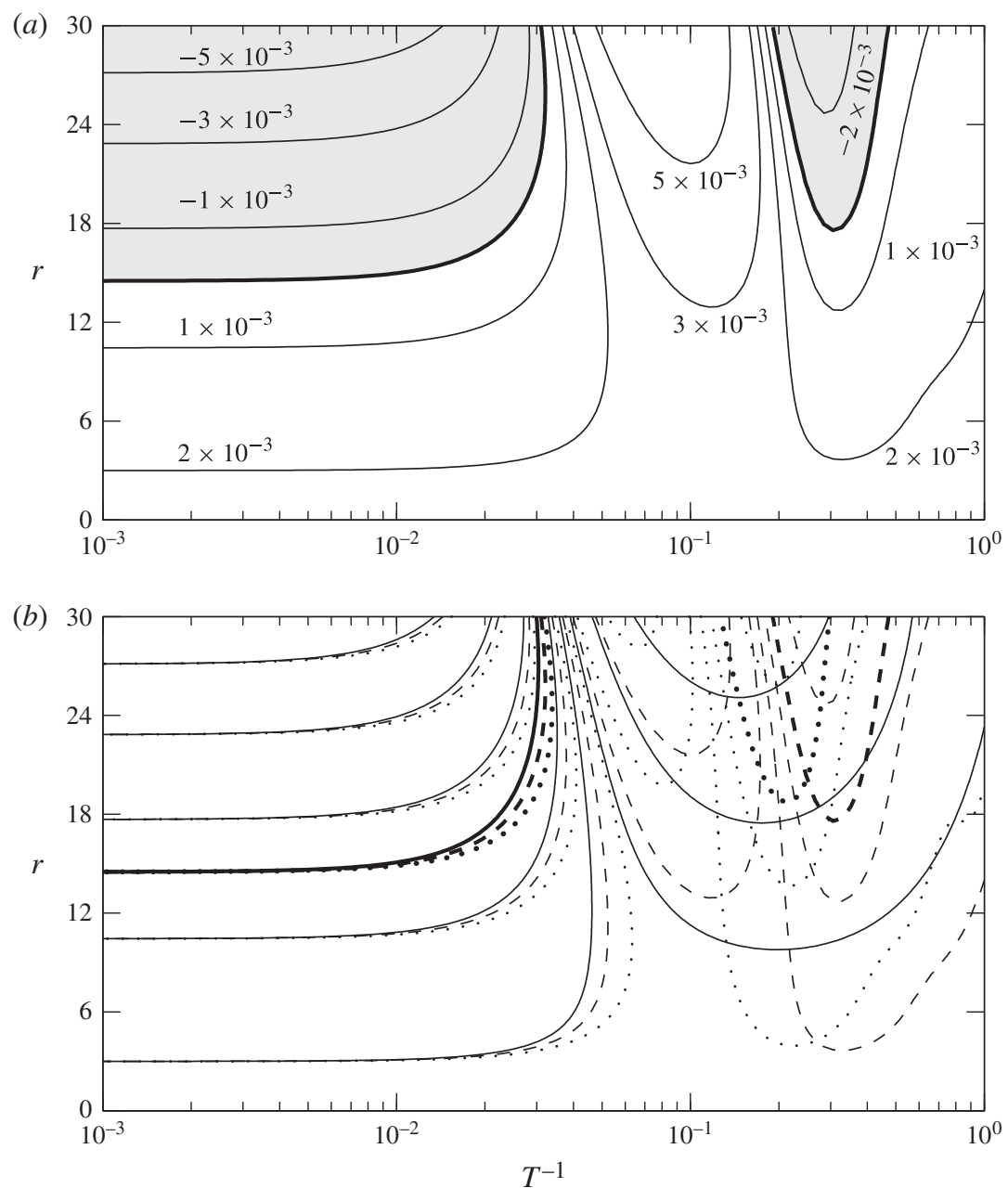

FIGURE 8. Influence of inertia on droplet transport as the period of oscillation, $T$, and the acceleration amplitude, $r$, vary. (a) Regime diagram using the same parameters as in figure 6 , but with $O h=5$. Note the secondary climbing regime present at small $T$ compared to figure $6(O h=0)$. (b) Overlaid contours from the regime diagrams in figure 6 (solid curves) and panel ( $a$ ) here (dashed curves), together with the regime diagram with $O h=10$ (dotted curves). Lower-frequency vibrations do not appear to be affected by the inclusion of weak inertial effects. The secondary climbing regime that is present at $O h=5$ is shifted towards lower frequencies for $O h=10$. The thicker curves demarcate the zero- $\bar{v}$ contours.

droplet width, $\Delta d(t)=d(t)-d(0)$, by solving the time-dependent problem with $d(0)=1.25$. The curve for $\ell(t)$ confirms that we have uphill motion, even though the droplet moves downhill initially, whereas the curve for $\Delta d(t)$ indicates that the initial transients due to spreading exist up to $t=O(10)$. Lastly, in figure $9(c)$ we show the portion of the regime diagram for $1 / 8 \leqslant T \leqslant 2$ and $50 \leqslant r \leqslant 150$ corresponding to figure 6 to demonstrate that, indeed, uphill motion is possible for $O h=0$ and at high frequencies. Admittedly, the range of values of $T$ that we considered is somewhat restricted, but it is important to emphasise that it becomes more likely for the droplet 
(a)
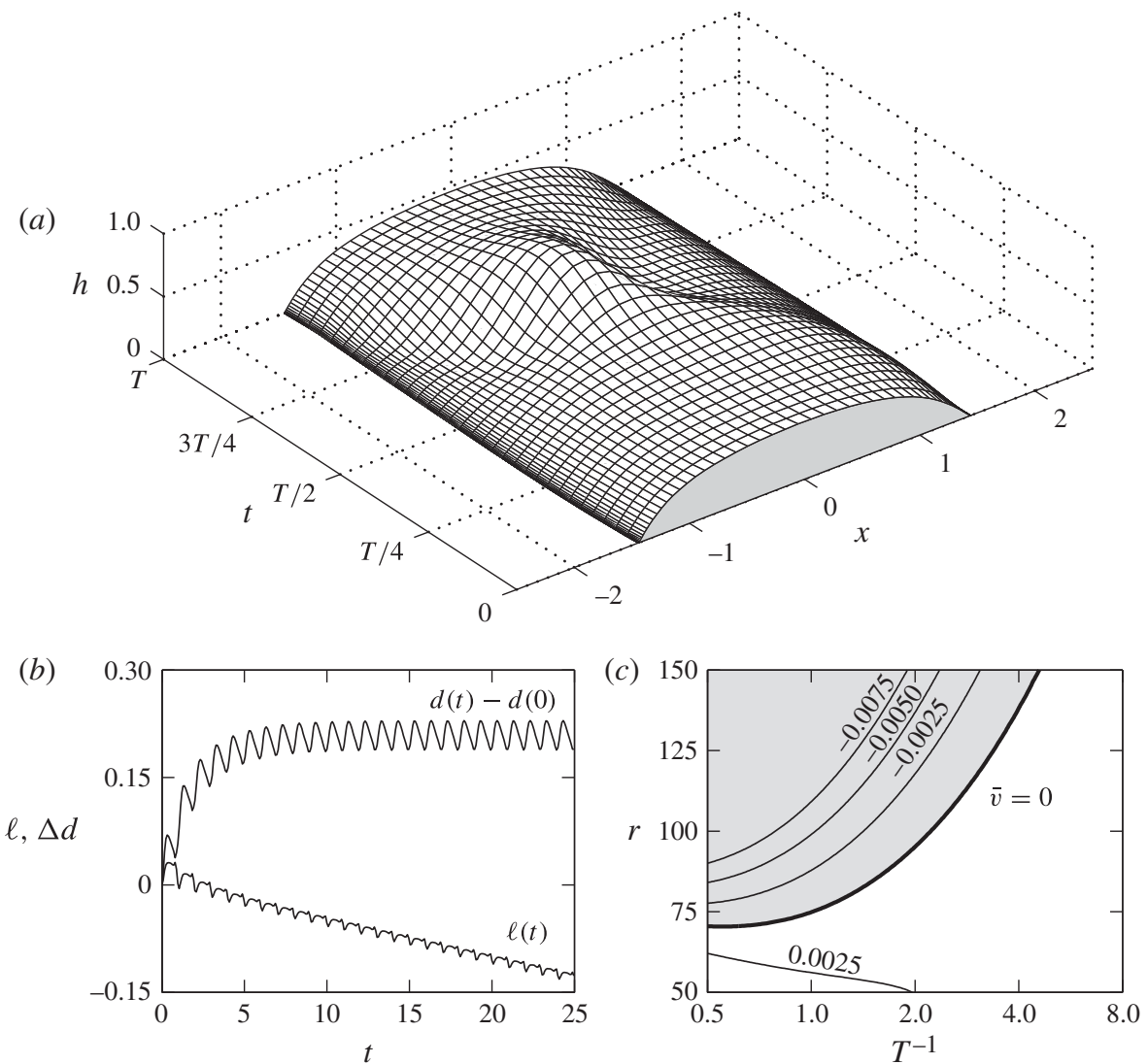

Figure 9. (a) Plot of $h(x, t)$ showing the long-time evolution of the droplet profile during a vibration period for an uphill-moving droplet with $T=1$ and $r=100$, keeping all other parameters the same as in figure $3(a)$. (b) Time-dependent computation corresponding to panel $(a)$ with $d(0)=1.25$, showing the evolution of $\ell(t)$ and $\Delta(t)=d(t)-d(0)$. (c) Portion of the $T^{-1}-r$ regime diagram shown in figure 6 at higher frequencies, where the shaded region represents the regime of uphill motion.

to experience instabilities and ultimately breakup if it undergoes accelerations of this magnitude for longer periods. Besides, under these conditions, the inclusion of inertia may be more appropriate.

\section{Heterogeneous substrates}

In the remainder of this study, we will look into the effects of substrate heterogeneities in greater detail. As previously mentioned, more focus will be placed on rough inclined substrates, but we will also comment briefly on the phenomenology of vibrated horizontal substrates.

\subsection{Horizontal substrates}

We saw in $\S 4.2$ that ideal substrates require symmetry breaking in order to induce net droplet transport to either the left or the right, which is achieved using a non-zero vibration angle, $\psi$. Symmetry breaking is also a prerequisite for net droplet motion 

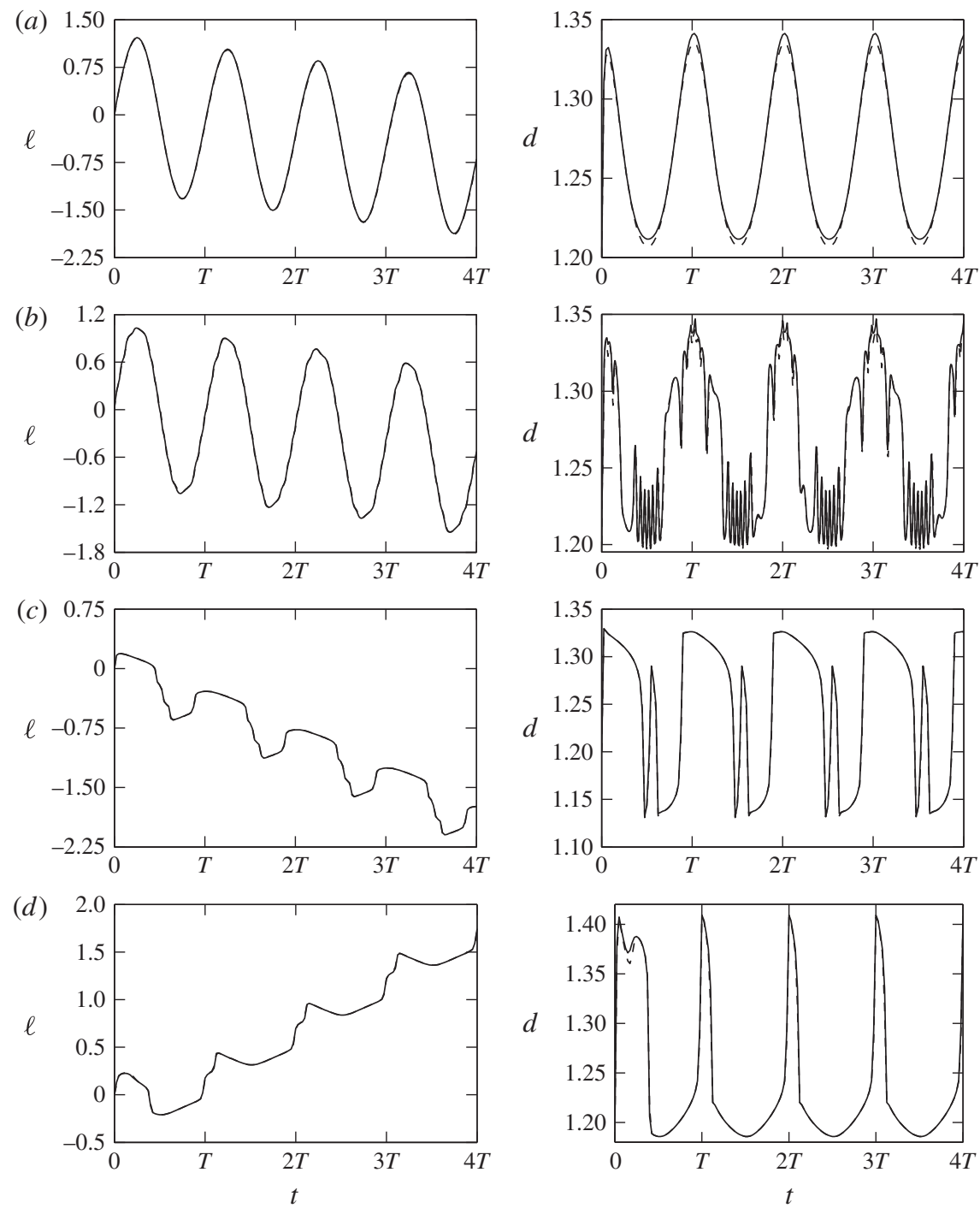

FIGURE 10. Influence of chemical heterogeneities on a droplet with $B o=0.5$ and $\alpha_{s}=10^{\circ}$ moving on a flat horizontal vibrating substrate with $r=2, \psi=5^{\circ}$ and $T=200$, when $b_{ \pm}(0)= \pm 1.2$. The evolution plots of $\ell(t)$ and $d(t)$ are shown for: $(a)$ the homogeneous case, $f(x)=1$; (b) a weak short-wavelength heterogeneity, $f(x)=1+0.1 \sin 20 x$; (c) a stronger heterogeneity, $f(x)=1+0.3 \sin 13 x$, which causes leftward droplet transport; $(d)$ a stronger heterogeneity, $f(x)=1+0.3 \sin 12 x$, which causes rightward motion. In all the panels, the solution to the PDE (solid curves), (2.5), and the IDEs obtained by matching (dashed curves), (3.23), are nearly indistinguishable.

in the presence of heterogeneities. However, the dynamics becomes considerably more complex, as the following set of computations illustrates.

In figure 10 we present examples of how chemical heterogeneities can influence the dynamics of vibrated horizontal substrates. In each of the four sets of calculations, all parameters are kept the same apart from the chemical heterogeneity function, $f(x)$, 
which prescribes the spatial contact angle variations. In all calculations, the vibration angle is set to $\psi=5^{\circ}$ to break the symmetry and allow for droplet transport (see figure caption for all parameters used).

In figure $10(a)$ the substrate is ideal and we see that $\ell(t)$ exhibits large-amplitude oscillations as the droplet moves to the left. The introduction of weak but rapid contact angle variations (see figure 10b) shows that, even though the evolution of $\ell(t)$ is not appreciably different from the ideal-substrate case, the droplet width exhibits $T$-periodic oscillations modulated by high-frequency oscillations. These secondary oscillations arise due to the moving fronts travelling across many wavelengths of the heterogeneity profile, which alter the contact line behaviour in order to accommodate the local variations in the equilibrium angle, noting that a similar effect was demonstrated for a rough substrate in figure $2(c)$. These seemingly high-frequency components do not invalidate the quasi-static assumption, as they occur on a long time scale, as also attested by the excellent agreement between our theory and solutions to the governing PDE.

Nevertheless, one can tune the heterogeneity function to get a more pronounced and less oscillatory motion compared to that in figure 10(a). For example, $f(x)$ is appropriately chosen in figure $10(c)$ to reduce the strong oscillations seen for $\ell(t)$ in figure $10(a)$ and to enhance the leftward motion. Heterogeneities, however, can also induce other types of motion. For instance, by merely changing the wavelength of the heterogeneity in figure 10(c), a different, unexpected behaviour is observed in figure $10(d)$ : the droplet moves to the right in spite of using a positive vibration angle, which naturally favours motion to the left. These calculations serve as an illustration of the intricate interplay of substrate heterogeneities and vibrations, whereby the appropriate choice of heterogeneities can possibly enhance or completely alter droplet transport. In contrast, such heterogeneities on non-vibrating substrates would only introduce barriers to motion.

The experiments of Duncombe et al. (2012a) have demonstrated that vertical vibrations of a horizontal ratcheted substrate can induce a unidirectional motion, thus suggesting that heterogeneities can also be used as another mechanism for breaking the symmetry in the system. Even though a detailed investigation of ratcheted substrates is beyond the scope of the present work, we have performed a few sample calculations to demonstrate that our model can also capture this behaviour.

In figure 11 we show a few examples based on a ratchet profile that was introduced in SK (see equation (6.14) in SK for its generic form). More specifically, we utilise the substrate profile $s(x)=s_{0} \zeta(x)$, where

$$
\zeta(x)=c \tanh (2 \sin 8 x) \ln \left[\frac{\cosh (2 \cos 4 x)}{\cosh (2 \sin 4 x)}\right],
$$

where $c$ is approximately equal to 1.1484 . The function $\zeta(x)$ produces a slightly sheared sine function with period $\pi / 8$ and amplitude 1 (see figure 11a). In figure $11(b)$ we show the case of a vertically vibrated drop with $r=6, T=100$, $B o=0.5$ and $\alpha_{s}=10^{\circ}$ moving on substrates of this form for three different $s_{0}$, namely $0.01,0.015$ and 0.02 . The largest-amplitude substrate, $s_{0}=0.02$, is able to trap the droplet, whereas droplets on substrates with $s_{0}=0.01$ and 0.015 behave in a very similar fashion, causing the droplet to be transported to the right. The picture is drastically altered when the vibration is made stronger, using $r=10$ (see figure 11c). When $s_{0}=0.015$ the rightward droplet motion is enhanced considerably. The same can be said for the $s_{0}=0.02$ substrate, for which the droplet also moves to the right at a 

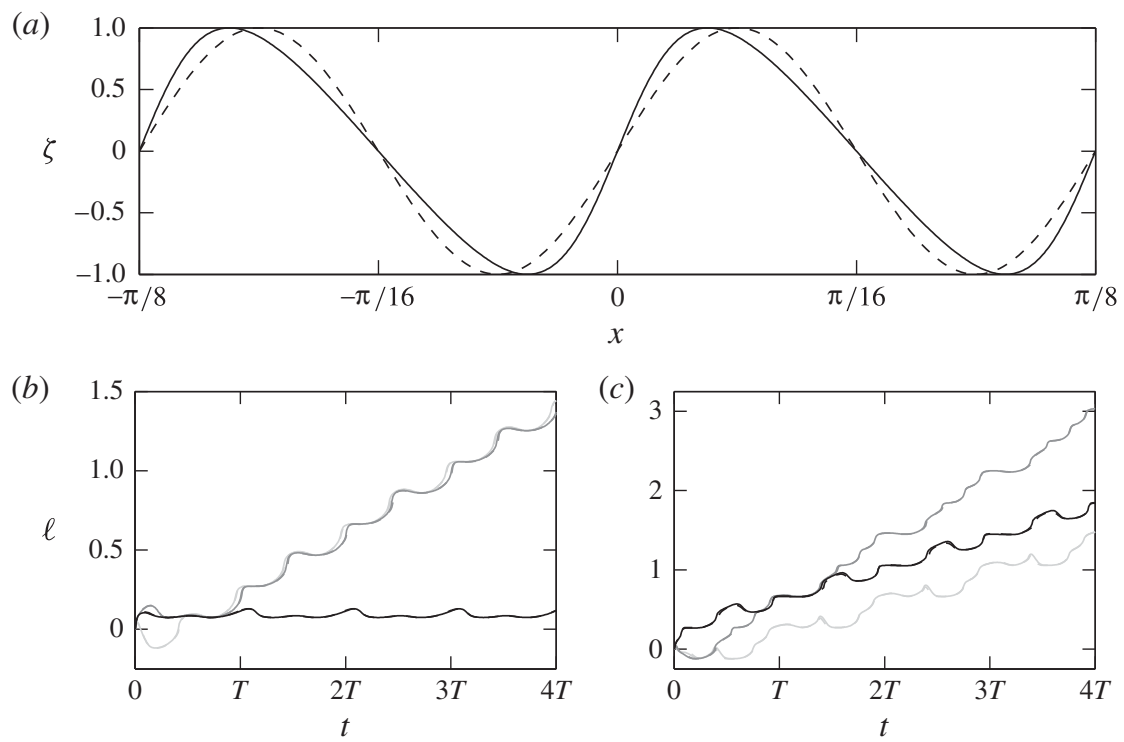

FIgURE 11. (a) Plot of $\zeta(x)$, (5.1) (solid curve), compared with the plot of sin $16 x$ (dashed curve). (b) Evolution of $\ell(t)$ corresponding to vertical vibrations with $r=6$ and $T=100$ of a horizontal rough substrate with profile $s(x)=s_{0} \zeta(x)$, where a drop, whose fronts were initially given by $b_{ \pm}(0)= \pm 1.1$, has $B o=0.5$ and $\alpha_{s}=10^{\circ}$. The black, dark grey and light grey curves correspond to $s_{0}=0.02,0.015$ and 0.01 , respectively. (c) As in panel $(b)$, but with $r=10$. Note that in panels $(b)$ and $(c)$ the solution to the PDE (solid curves) and the IDEs obtained by matching (dashed curves) are indistinguishable.

rate that is faster than for the lowest-amplitude substrate, $s_{0}=0.01$. This remarkable result is a prime example that illustrates that higher-amplitude substrates can enhance droplet transport more than lower-amplitude ones, but this strongly depends on the droplet and vibration characteristics. We will return to this towards the end of the next section, where we compute a regime diagram that shows the dependence of $\bar{v}$ on the amplitude and wavelength of a rough inclined substrate.

\subsection{Inclined substrates}

Obtaining a regime diagram like the one shown in figure 6 is considerably more difficult numerically when substrate heterogeneities are present. This is because we can no longer use the technique employed in figure 6, where we assumed that $h(x, t), \dot{\ell}(t)$ and $\dot{d}(t)$ are $T$-periodic. The reason is because substrate heterogeneities can sometimes yield behaviours that are periodic with a different period. This is shown in figure $11(a-d)$, where we present calculations of a rough substrate whose profile is given by $s(x)=0.02 \cos 9.2 x$ with $\phi=2^{\circ}, \psi=10^{\circ}, \alpha_{s}=15^{\circ}, B o=0.1$ and $T=100$ for different values of $r$. More specifically, we plot $d$ as a function of time modulo the period of oscillation, as an efficient way to detect the period of the motion, which is found by the number of distinct curves times the vibration period, $T$. Hence, seeing a single distinct curve, where the rest of the data collapses onto this curve, corresponds to $T$-periodic motion, two curves corresponds to $2 T$-periodic motion, etc. In all the plots in figure 12 , we show the results for $5 T \leqslant t \leqslant 55 T$, having neglected the first five vibration periods to avoid the transient effects. We 

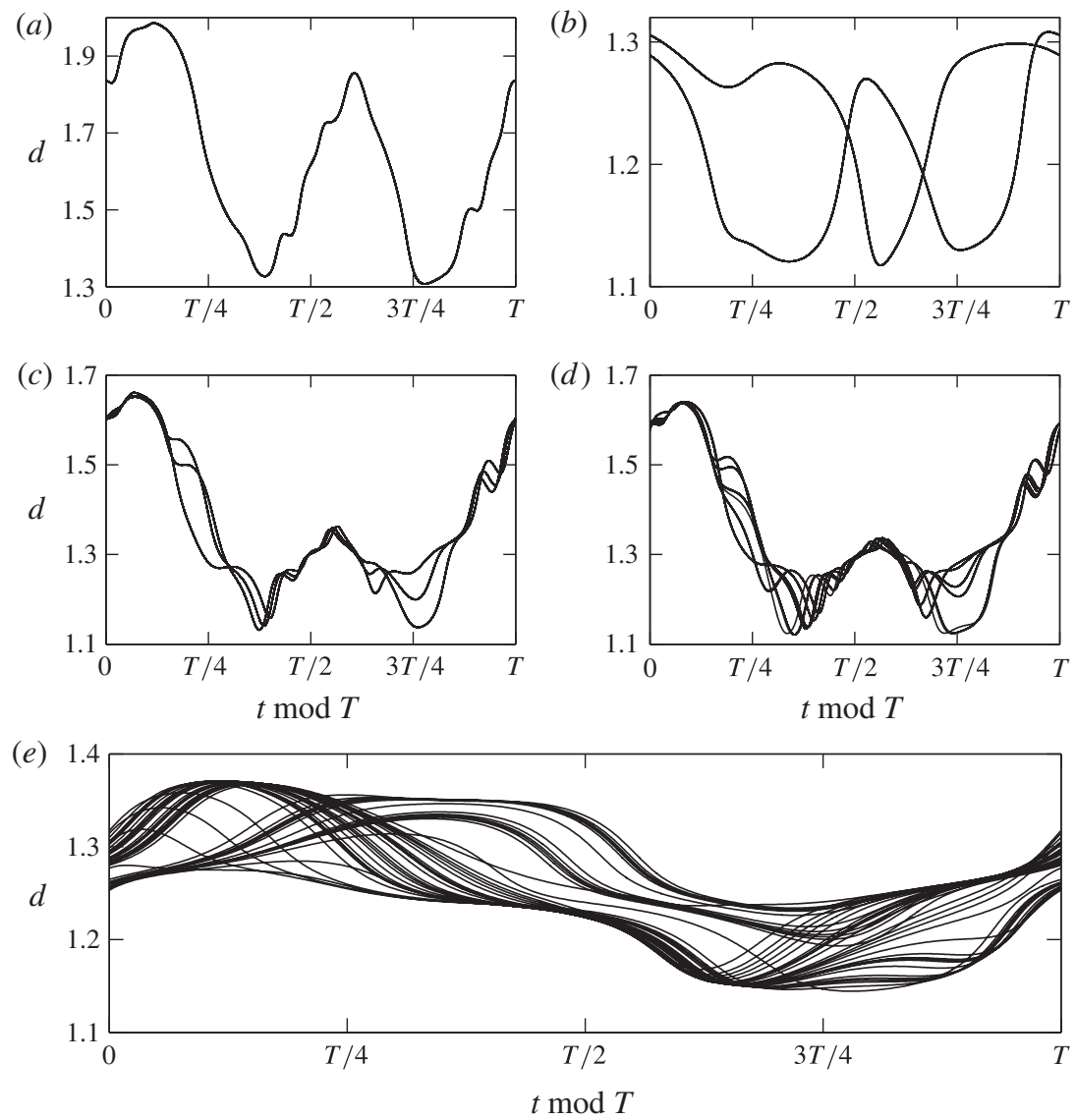

FIGURE 12. Evolution of $d$ as a function of time modulo the period of oscillation plotted for $5 T \leqslant t \leqslant 55 T$. (a) $T$-periodic evolution with parameters $s(x)=0.02 \cos 9.2 x$ with $\phi=2^{\circ}$, $\psi=10^{\circ}, \alpha_{s}=15^{\circ}, B o=0.1$ and $T=100$. (b) As in panel $(a)$, with $r=6$ (2T-periodic evolution). (c) As in panel (a), with $r=22.5$ (3T-periodic evolution). (d) As in panel $(a)$, with $r=22$ (6T-periodic evolution). (e) Plot corresponding to the calculation shown in figure $2(d)$. No period could be identified.

observe that, when $r=30$, the motion is $T$-periodic (figure $12 a$ ), when $r=6$ it is $2 T$-periodic (figure $12 b$ ), when $r=22.5$ it is $3 T$-periodic (figure $12 c$ ) and when $r=22$ the free-surface oscillations are $6 T$-periodic. In figure $12(e)$, which corresponds to the calculation shown in figure $12(d)$, the motion appears to be quasi-periodic, since no period could be detected, at least over the duration of the simulation.

The period of free-surface oscillations cannot be known a priori and the previously used numerical technique no longer applies. To partially remedy this difficulty, we solve (2.5) forward in time for a specified number of vibration periods, neglect the transient dynamics and extract an approximation for $\bar{v}$ based on the remaining data. This is a time-consuming process and often involves many thousands of such calculations to reveal a portion of the regime diagram. To construct each of the following $T^{-1}-r$ regime diagrams, we have used this method and performed 18750 simulations on a $150 \times 125$ grid in the $T^{-1}-r$ parameter space, bearing in mind that 
an accurate estimate of $\bar{v}$ is possible only if a large number of periods are taken into account. For this calculation, we considered 20 periods, after discarding any transient effects to avoid including, for example, the regime where spreading effects are dominant.

Instead of a contour plot shown, for example, in figures 6 and $8(a)$, we have filled the areas between contours with different shades of grey depending on the sign of $\bar{v}$. In an attempt to represent both negative and positive values of $\bar{v}$ using greyscale shades, we mapped in a discontinuous manner each range of values of $\bar{v}$ to a certain grey shade so that darker shades correspond to uphill motion and lighter shades to downhill motion (see greyscale bar in figure 13d). Within the unshaded white regions, the droplet moves slowly with $|\bar{v}| \leqslant 10^{-3}$. Given that the computation of the $\bar{v}=0$ curve is more sensitive to the number of oscillations considered in the estimation of $\bar{v}$, this curve is not shown, with the understanding that in the case of rough surfaces there are regions within the unshaded areas where the droplet is trapped by the features of the heterogeneities with no net transport.

In all the calculations shown in figure 13, we have used the same parameters as in the ideal-substrate case in figure 6. Moreover, in order to make comparisons with the regime diagrams of the ideal-substrate case, we have reproduced figure 6 with the aforementioned greyscale scheme (see figure 13a). As the substrate is made rough (see figure $13 b$, where we used $s(x)=0.01 \cos 20 x$ ), the unshaded regions expand, which is suggestive of the fact that roughness tries to prevent the downhill motion. In fact, nearly throughout region $B$ the droplet has practically zero mean speed compared to region $A$ for which we get nearly zero speed only within a portion of the corresponding parameter space. By doubling the amplitude of the substrate heterogeneity (see figure $13 c$, where we used $s(x)=0.02 \cos 20 x$ ), we see further expansion of the unshaded, slow-transport regions. It is also interesting to note that the region over which we get uphill motion is not appreciably altered by weak roughness, recalling that a similar observation was made in $\S 4.4$, while considering weak inertial effects. These calculations seem to suggest that the so-called 'static' regions observed in the experiments of Brunet et al. (2007, 2009), where the droplet does not exhibit net transport, can also possibly be attributed, at least in part, to unavoidable substrate heterogeneities that are naturally present.

An interesting problem arising from these considerations is finding the optimal substrate amplitude and wavelength that maximise $|\bar{v}|$ for fixed vibration and droplet characteristics. However, solving the associated optimisation problem is beyond the scope of the present work, and a brute-force computation was used to obtain figure 14, namely by solving the associated PDE thousands of times and determining $\bar{v}$ over 10 oscillation periods, having neglected the initial transient dynamics.

In figure 14 we show contours of $\bar{v}$ as the amplitude, $s_{0}$, and wavenumber, $k_{0}$, are varied for a substrate whose profile is given by $s(x)=s_{0} \cos k_{0} x$ (see figure caption for the remaining parameters). As expected, we find that, as the substrate becomes more rough (larger $s_{0}$ and $k_{0}$ ), no net motion and reduced transport are favoured. Most importantly, note the alternating regions in the parameter space of enhanced uphill and downhill motion, which is especially pronounced at moderately small $k_{0}$. Noteworthy also is that, even though one might have inferred from previous regime diagrams throughout this work that vibrations with $r=6$ might be too weak for uphill motion to occur, we see that by appropriately tuning the substrate properties this becomes feasible.

Interestingly, there are important structural changes in the associated regime diagrams for substrates that optimise droplet transport. These are shown in figure 15, 

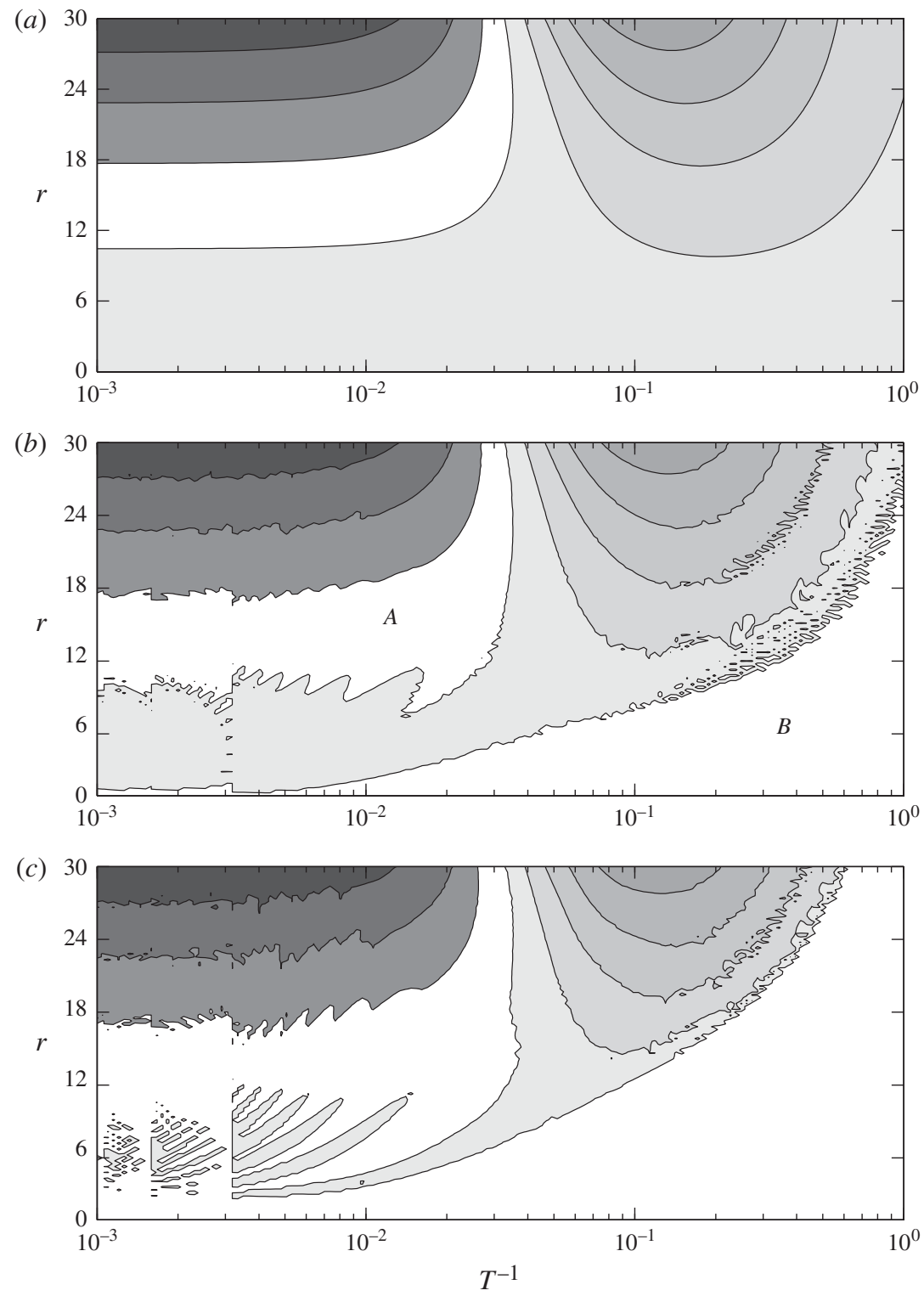

(d)

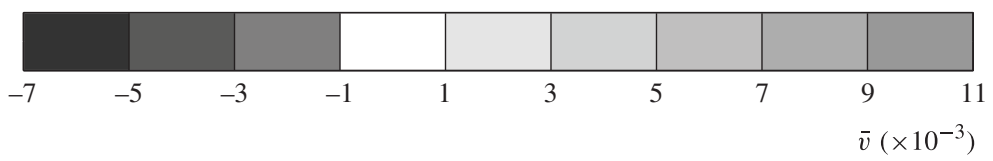

FIGURE 13. $(a-c)$ Regime diagrams showing the dependence on substrate heterogeneities. Areas between contours of $\bar{v}$ are shaded according to the greyscale bar in panel $(d)$. In particular, panel $(a)$ is a reproduction of the regime diagram of figure 6 ; in panels $(b)$ and $(c)$ the same parameters are used as in panel $(a)$, but with $s(x)=0.001 \sin 20 x$ and $s(x)=0.002 \sin 20 x$, respectively. (d) Greyscale bar for the areas between the $\bar{v}$ contours in panels $(a-c)$. Darker shades of grey correspond to uphill motion and lighter shades to downhill motion; the unshaded regions correspond to $|\bar{v}| \leqslant 10^{-3}$. 


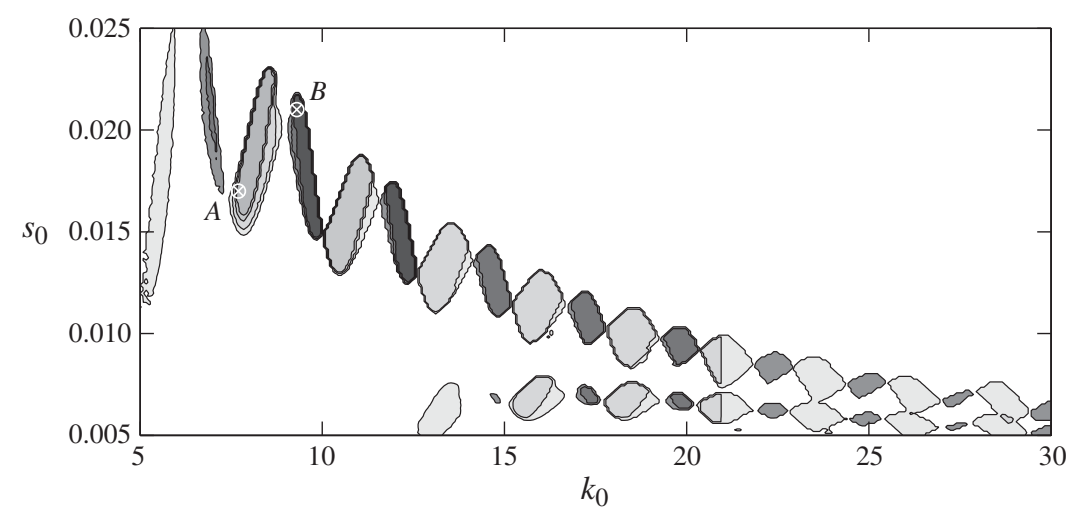

FIGURE 14. Influence of substrate topography on droplet transport, showing contours of $\bar{v}$ as the substrate wavenumber, $k_{0}$, and amplitude, $s_{0}$, vary for substrate profiles given by $s(x)=s_{0} \cos k_{0} x$. Areas between the contours of $\bar{v}$ are filled according to the greyscale bar in figure $13(d)$ (lighter grey regions correspond to downhill motion; darker grey regions to uphill motion; in the unshaded regions the droplet speed is typically very small). The remaining parameters used in the computation are $\lambda=0.005, B o=0.1, T=100, r=6$, $\phi=2^{\circ}, \psi=10^{\circ}$ and $\theta=15^{\circ}$. Point $A$, for which $k_{0}=7.7$ and $s_{0}=0.017$, lies in the vicinity of optimal downhill motion, whereas point $B$, for which $k_{0}=9.3$ and $s_{0}=0.021$, lies in the vicinity of optimal uphill motion (see also figure 15 for the corresponding regime diagrams).

where we fix $s_{0}$ and $k_{0}$ appropriately and let $r$ and $T$ vary, while keeping the other parameters as in figure 14. For comparison, we show in figure 15(a) the $r-T^{-1}$ regime diagram in the absence of substrate topography, where we see that for $r=6$ and $T=100$ the droplet translates with a small downhill speed. The calculation is repeated in figure $15(b)$ with substrate parameters that lie in the vicinity of optimal downhill motion when $r=6$ and $T=100\left(s_{0}=0.017, k_{0}=7.7\right.$; see point $A$ in figure 14). We observe that generally downhill regions are significantly diminished, yet we see that downhill motion is enhanced more than 10 -fold at $r=6$ and $T=100$ compared to figure $15(a)$.

On the other hand, choosing substrate parameters that lie near the optimal uphill motion when $r=6$ and $T=100\left(s_{0}=0.021\right.$ and $k_{0}=9.3$; see point $B$ in figure 14), the regime diagram in figure $15(c)$ reveals a markedly different behaviour. In comparison with other regime diagrams shown throughout this study, the droplet can no longer move downhill at lower frequencies and we only get uphill motion when $r$ is sufficiently high. This is another illustration that highlights the fact that substrate heterogeneities, no matter how small, can profoundly affect the underlying droplet dynamics when appropriately chosen. Moreover, this optimisation of substrate parameters can be of great importance from a practical point of view, especially in applications where it is desirable to enhance unidirectional droplet transport using weaker vibrations.

\subsection{Hysteresis-like effects}

We have seen throughout this study that one of the features introduced by substrate heterogeneities is their ability to trap the contact line and prevent the droplet from exhibiting net transport (see e.g. figure $2 b$ ). Even though our model does 

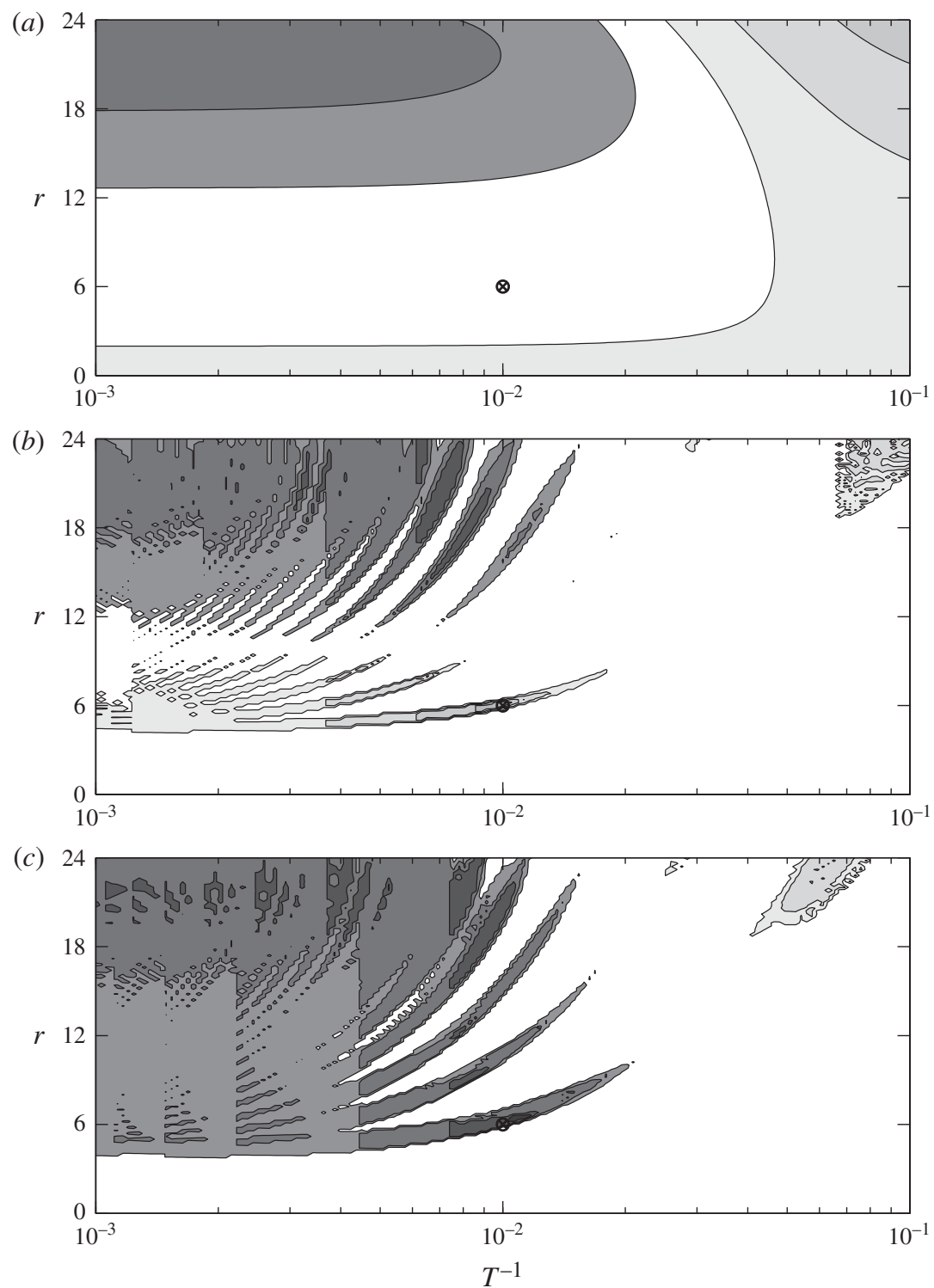

FIgURE 15. Regime diagrams based on the calculation in figure 14 by allowing $r$ and $T$ to vary for fixed substrate profiles. The points demarcated with a crossed circle correspond to $r=6, T=100$, which directly correspond to parameters in figure 14. (a) Ideal-substrate case, $s_{0}=0$; at the crossed circle, $\bar{v}=0.0006$. (b) Rough substrate with $s_{0}=0.017$ and $k_{0}=7.7$. The crossed circle corresponds to point $A$ in figure $14(\bar{v}=0.0082)$. (c) Rough substrate with $s_{0}=0.021$ and $k_{0}=9.3$. The crossed circle corresponds to point $B$ in figure $14(\bar{v}=-0.0068)$.

not incorporate the effects of contact angle hysteresis by prescribing advancing and receding contact angles, which would pin the droplet fronts, the use of heterogeneities can yield hysteresis-like effects. Besides, we also ascribe to the view that heterogeneities are the principal source of contact angle hysteresis (see 

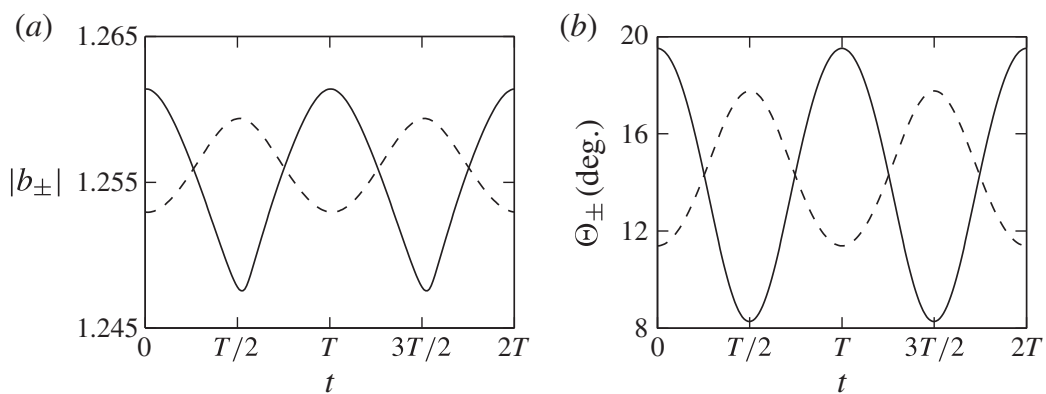

(c)

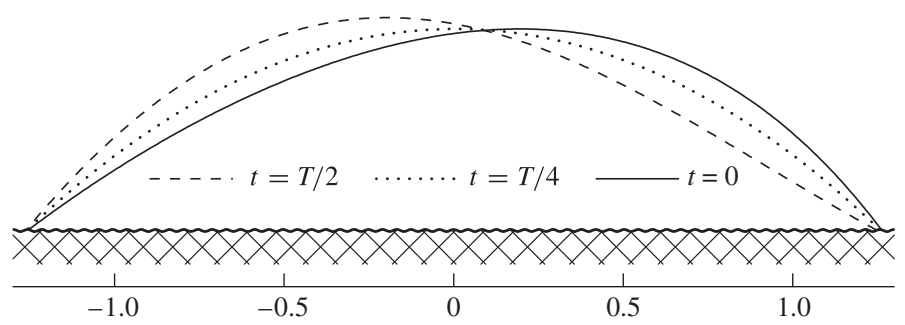

FIGURE 16. Trapped droplet dynamics computed using the IDEs obtained by matching, (3.23). The same parameters are used as in figure 2(a), but with $s(x)=0.003 \cos 150 x$ and $r=9$. (a) Evolution of the location of the two droplet fronts. (b) Evolution of the dimensional apparent contact angles, computed as if the substrate were ideally flat, using (3.11) with $s(x)=0$. In panels $(a)$ and $(b)$ the solid and dashed curves refer to the evolutions for the right and left droplet fronts, respectively. (c) Shapes of the free surface using the leading-order outer solution, namely plots of $h_{0}+s$ as a function of $x=d y+\ell$, at different times. When $t=0$ and $t=T / 2$ the droplet shape exhibits the largest deformations. The substrate shape is also shown for clarity.

also discussion on non-vibrating droplets on inclined substrates (Savva \& Kalliadasis 2013)).

Noting that the amplitude of the oscillations of the fronts can be diminished by using a rougher substrate, we show in figure 16 the results of such a calculation with a small-amplitude short-wavelength substrate whose profile is given by $s(x)=0.003 \cos 150 x$, using the equations obtained by matching, (3.23) (see figure caption for the parameters used). For a droplet with characteristic length scale $L=1 \mathrm{~mm}$, the amplitude and wavelength of the topography of such a substrate would be approximately $0.8 \mu \mathrm{m}$ and $42 \mu \mathrm{m}$, respectively. In figure 16(a) we plot the evolution of the droplet fronts over two periods of oscillation after discarding the initial transient dynamics. We observe that the droplet fronts are not actually pinned, but exhibit small-amplitude oscillations. However, macroscopically the droplet appears to be pinned with oscillating contact angles. This is illustrated in figure 16(c), where we show the leading-order free-surface shape at the times it exhibits the largest deformations $(t=0$ and $t=T / 2)$. The oscillating angles are shown in figure 16(b), where we plot the apparent contact angles $\Theta_{ \pm}$corresponding to the two fronts at $x=b_{ \pm}$, which are computed as if the substrate were ideally flat, using (3.11) with $s(x)=0$ and the reference angle $\alpha_{s}=15^{\circ}$. Clearly, substrate profiles of this kind can be thought of as more representative of real surfaces than the ones we have used in the preceding sections, but it is to be noted that solving the governing PDE becomes increasingly more difficult as we decrease the wavelength of the topography. 


\section{Concluding remarks}

In the present study, we considered how the combined effects of gravity, substrate vibrations and substrate heterogeneities influence the dynamics of 2D droplets, by adopting the appropriate long-wave models. By assuming that the dynamics is quasi-static, we have utilised the method of matched asymptotic expansions to deduce a set of IDEs for the evolution of the two droplet fronts in the limit of long oscillation periods. This analysis, which was verified by numerous numerical experiments performed throughout this work, was largely based on the recent work of the present authors on inclined heterogeneous substrates (Savva \& Kalliadasis 2013). It is important to emphasise that, even though we assumed the presence of slip in our model, our results can be easily mapped to other types of models that are commonly used to relax the stress singularity occurring at a moving contact line (see e.g. Savva \& Kalliadasis 2011; Sibley, Savva \& Kalliadasis 2012; Sibley et al. 2013a,b).

In the limit of low-frequency vibrations $\left(T^{-1} \rightarrow 0\right)$, we obtained an expression for the mean drift velocity, (4.4), valid for small $B o, \phi$ and $\psi$. From this calculation, we were able to deduce a climbing criterion, (4.6), so that, by vibrating the substrate sufficiently strongly, uphill motion can be observed. This result and the regime diagrams we obtained are qualitatively consistent with the numerics conducted by John \& Thiele (2010) with precursor film models and the analytical work of Benilov \& Cummins (2013), but for 3D droplets with large contact angles. In contrast, related analyses with $2 \mathrm{D}$ droplets predicted vanishing $\bar{v}$ as $T^{-1} \rightarrow 0$ (Benilov 2011; Benilov \& Billingham 2011), thus implying that climbing cannot be observed in the low-frequency limit.

We have also briefly considered the inclusion of weak inertial effects, observing appreciable changes in the high-frequency limit, such as the reduced average speeds and the presence of a secondary climbing regime, which is likely to be the regime where the experiments by Brunet et al. (2007, 2009) were conducted. This secondary climbing regime was also shown to be present in the inertia-less case as well, but droplets would need to undergo strong vibrations at high frequencies, which could make them more susceptible to instabilities. Moreover, the 'static' parameter regimes, i.e. the regimes where no net transport is observed, which typically occur at higher frequencies, may be possibly be attributed, at least partly, to inertial effects.

In our view, to rationalise the 'static' regimes, it might be necessary also to include the substrate heterogeneities, which manifest themselves macroscopically as contact angle hysteresis. Even though our model does not assume hysteresis, by prescribing, for example, advancing and receding contact angles, it can exhibit hysteresis-like effects if the heterogeneities vary at smaller length scales. We have shown that substrate heterogeneities influence the underlying dynamics quite significantly at both low and high frequencies. In fact, we have demonstrated for the first time the rich behaviour of this system, whereby heterogeneities can enhance and impair droplet transport, both uphill and downhill, if appropriately tuned. We have also seen that directed droplet transport on vertically vibrated horizontal substrates is also possible by using ratcheted substrates, which is qualitatively consistent with the experiments of Duncombe et al. $(2012 a, b)$. Admittedly, we have only considered somewhat contrived heterogeneity profiles, instead of, say, heterogeneities that take into account random effects that are naturally present in a real substrate (see e.g. Savva, Kalliadasis \& Pavliotis 2010; Savva, Pavliotis \& Kalliadasis 2011a,b), but we chose to do so since the principal focus of the present work was to highlight the most important features of the possible dynamic scenarios in the many-dimensional parameter space of the problem. 
Since we have observed uphill motion without the need for contact angle hysteresis, our model supports the mechanism proposed by John \& Thiele (2010), who argued that the observed uphill motion is due to the vertical vibrations of the substrate, which ultimately influence the lateral motion of the droplet through the deformations caused on the free surface of the droplet. This, however, can only occur provided that the substrate is vibrated in such a way that breaks the back-and-forth symmetry of the lateral motion. For example, a droplet on an inclined substrate can never move uphill if $\psi=0^{\circ}$, regardless of the strength of vibrations. In this case, one can utilise a different mechanism to break the symmetry and yield directed transport, such as the heterogeneities of the substrate.

Lastly, we should note that the results of the present work are not directly verifiable with existing experiments (see e.g. Noblin, Buguin \& Brochard-Wyart 2004; Brunet et al. 2007, 2009; Noblin et al. 2009; Duncombe et al. 2012a,b), mainly as a result of the 2D geometry considered, which not only makes the droplet susceptible to transverse instabilities, but also requires that the substrate be vibrated more strongly to get the droplet to climb uphill (see also John \& Thiele 2010; Benilov 2011). However, the main qualitative features of the dynamics is adequately captured with our model, and the present study can be viewed as a first step towards gaining a better physical understanding of the phenomenon. Thus, to allow for more reliable quantitative comparisons with experiments, the combined effects of substrate heterogeneities, inertia and the three-dimensionality of drops need to be considered, a considerably more difficult problem to be examined both analytically and numerically.

\section{Appendix A. Derivation of (4.7)}

Using the scales introduced in the text, we use as a starting point the nondimensional Navier-Stokes equations,

$$
\begin{gathered}
O h\left\{\partial_{t} u+\partial_{x}\left(u^{2}\right)+\partial_{z}(u v)\right\}=-\partial_{x} p+\frac{1}{3} \partial_{z}^{2} u+w_{2}, \\
0=-\partial_{z} p-w_{1},
\end{gathered}
$$

as simplified in a long-wave expansion, where $u$ and $v$ are the velocities in the $x$ and $z$ directions, respectively, and $p$ is the pressure. The momentum equations are supplemented with the continuity equation

$$
\partial_{x} u+\partial_{z} v=0
$$

and boundary conditions at the free surface of the droplet $(z=h)$ and the substrate $(z=0)$. More specifically, at $z=h$ we have the kinematic boundary condition

$$
\partial_{t} h+u \partial_{x} h=v,
$$

together with the normal and tangential stress balance,

$$
p=-\partial_{x}^{2} h \quad \text { and } \quad \partial_{z} u=0,
$$

respectively. At $z=0$, we have the slip and no-penetration conditions,

$$
u=\frac{1}{3} \lambda \partial_{z} u \text { and } v=0,
$$

respectively. The pressure can be readily computed from (A 2) and (A 4b), and we find

$$
p=-\partial_{x}^{2} h+w_{1}(h-z) .
$$


Next, consider the integral of (A 1 ) with respect to $z$ from 0 to $h$, which simplifies to

$$
O h\left\{\partial_{t} \int_{0}^{h} u \mathrm{~d} z+\partial_{x}\left(\int_{0}^{h} u^{2} \mathrm{~d} z\right)\right\}=h\left(\partial_{x}^{3} h-w_{1} \partial_{x} h+w_{2}\right)-\left.\frac{1}{3} \partial_{z} u\right|_{z=0}
$$

by using (A 5) and applying conditions (A 4a), (A 4c) and (A 4e) to eliminate the boundary terms. To proceed, we assume that the velocity profile is parabolic in the $z$ direction,

$$
u=\left(\frac{1}{2} z^{2}-z h-\frac{1}{3} \lambda h\right) c(x, t),
$$

which automatically satisfies conditions (A $4 c$ ) and (A 4e). Letting $q(x, t)$ denote the flow rate in the $x$ direction,

$$
q=\int_{0}^{h} u \mathrm{~d} z
$$

we find that

$$
c=-\frac{3 q}{h^{2}(h+\lambda)} .
$$

Then, by substituting the profile (A 7) in (A 6) and making use of (A 9), it is a matter of simple algebra to obtain (4.7a).

Equation (4.7b) corresponds to mass conservation. It can be obtained by integrating (A 3) with respect to $z$ from 0 to $h$ to obtain $\left.v\right|_{z=h}$, which is then substituted into the kinematic boundary condition, (A 4) to yield (4.7b).

\section{REFERENCES}

Benilov, E. S. 2010 Drops climbing uphill on a slowly oscillating substrate. Phys. Rev. E 82, 026320.

Benilov, E. S. 2011 Thin three-dimensional drops on a slowly oscillating substrate. Phys. Rev. E 84, 066301.

Benilov, E. S. \& Billingham, J. 2011 Drops climbing uphill on an oscillating substrate. J. Fluid Mech. 674, 93-119.

Benilov, E. S. \& Cummins, C. P. 2013 Thick drops on a slowly oscillating substrate. Phys. Rev. E 88, 023013.

Bonn, D., Eggers, J., Indekeu, J., Meunier, J. \& Rolley, E. 2009 Wetting and spreading. Rev. Mod. Phys. 81, 739-805.

Bormashenko, E., Pogreb, R., Stein, T., Whyman, G., Erlich, M., Musin, A., Machavariani, V. \& AURBACH, D. 2008 Characterization of rough substrates with vibrated drops. Phys. Chem. Chem. Phys. 10, 4056-4061.

Bormashenko, E., Pogreb, R., Whyman, G., Bormashenko, Y. \& Erlich, M. 2007a Vibrationinduced Cassie-Wenzel wetting transition on rough surfaces. Appl. Phys. Lett. 90, 201917.

Bormashenko, E., Pogreb, R., Whyman, G. \& Erlich, M. 2007b Cassie-Wenzel wetting transition in vibrating drops deposited on rough surfaces: Is the dynamic Cassie-Wenzel wetting transition a 2D or 1D affair? Langmuir 23, 6501-6503.

Bormashenko, E., Pogreb, R., Whyman, G. \& Erlich, M. 2007c Resonance Cassie-Wenzel transition for horizontally vibrated drops deposited on a rough surface. Langmuir 23, 12217-12221.

Bostwick, J. B. \& Steen, P. H. 2009 Capillary oscillations of a constrained liquid drop. Phys. Fluids 21, 032108.

Brunet, P., Eggers, J. \& Deegan, R. D. 2007 Vibration-induced climbing of drops. Phys. Rev. Lett. 99, 144501.

Brunet, P., Eggers, J. \& Deegan, R. D. 2009 Motion of a drop driven by substrate vibrations. Eur. Phys. J., Spec. Top. 166, 11-14. 
Celestini, F. \& Kofman, R. 2006 Vibration of submillimeter-size supported droplets. Phys. Rev. E 73, 041602.

Daniel, S. \& Chaudhury, M. K. 2002 Rectified motion of liquid drops on gradient surfaces induced by vibration. Langmuir 18, 3404-3407.

Daniel, S., Chaudhury, M. K. \& De Gennes, P.-G. 2005 Vibration-actuated drop motion on surfaces for batch microfluidic processes. Langmuir 21, 4240-4248.

Daniel, S., Sircar, S., Gliem, J. \& Chaudhury, M. K. 2004 Ratcheting motion of liquid drops on gradient surfaces. Langmuir 20, 4085-4092.

Della Volpe, C., Maniglio, D., Morra, M. \& Siboni, S. 2002 The determination of a 'stableequilibrium' contact angle on heterogeneous and rough surfaces. Colloids Surf. A 206, 47-67.

Dong, L., Chaudhury, A. \& Chaudhury, M. K. 2006 Lateral vibration of a water drop and its motion on a vibrating surface. Eur. Phys. J. E 21, 231-242.

Duncombe, T. A., Erdem, E. Y., Shastry, A., Baskaran, R. \& Böhringer, K. F. $2012 a$ Controlling liquid drops with texture ratchets. Adv. Mater. 24, 1545-1550.

Duncombe, T. A., Parsons, J. F. \& Böhringer, K. F. $2012 b$ Directed drop transport rectified from orthogonal vibrations via a flat wetting barrier ratchet. Langmuir 28, 13765-13770.

Fayzrakhmanova, I. S. \& Straube, A. V. 2009 Stick-slip dynamics of an oscillated sessile drop. Phys. Fluids 21, 072104.

Hocking, L. M. 1983 The spreading of a thin drop by gravity and capillarity. Q. J. Mech. Appl. Maths 36 (1), 55-69.

Hocking, L. M. 1992 Rival contact-angle models and the spreading of drops. J. Fluid Mech. 239, $671-681$.

James, A. J., Vukasinovic, B., Smith, M. K. \& Glezer, A. 2003 Vibration-induced drop atomization and bursting. J. Fluid Mech. 476, 1-28.

John, K. \& Thiele, U. 2010 Self-ratcheting Stokes drops driven by oblique vibrations. Phys. Rev. Lett. 104, 107801.

Johnson, R. E. \& Dettre, R. H. 1964 Contact angle hysteresis I. Study of an idealized rough surface. Adv. Chem. Ser. 43, 112-135.

KELVIN, LORD 1863 Dynamical problems regarding elastic spherodial shells and speroids of incompressible liquid. Phil. Trans. R. Soc. Lond. 153, 583-616.

Lamb, M. A. 1881 On the oscillations of a viscous spheroid. Proc. Lond. Math. Soc. 13, 51-70.

LóPeZ, P. G., Miksis, M. J. \& BAnkoff, S. G. 1997 Inertial effects on contact line instability in the coating of a dry inclined plate. Phys. Fluids 9 (8), 2117-2183.

Lyubimov, D. V., Lyubimova, T. P. \& Shklyaev, S. V. 2006 Behavior of a drop on an oscillating solid plate. Phys. Fluids 18, 012101.

Meiron, T. S., Marmur, A. \& Saguy, I. S. 2004 Contact angle measurement on rough surfaces. J. Colloid Interface Sci. 274, 637-644.

Mettu, S. \& Chaudhury, M. K. 2010 Stochastic relaxation of the contact line of a water drop on a solid substrate subjected to white noise vibration: roles of hysteresis. Langmuir 26 (11), $8131-8140$.

Mettu, S. \& Chaudhury, M. K. 2011 Motion of liquid drops on surfaces induced by asymmetric vibration: role of contact angle hysteresis. Langmuir 27, 10327-10333.

Noblin, X., Buguin, A. \& Brochard-Wyart, F. 2004 Vibrated sessile drops: transition between pinned and mobile contact line oscillations. Eur. Phys. J. E 14, 395-404.

Noblin, X., Kofman, R. \& Celestini, F. 2009 Ratchetlike motion of a shaken drop. Phys. Rev. Lett. 102, 194504.

RaYleigh, Lord 1879 On the capillary phenomena of jets. Proc. R. Soc. Lond. 29, 71-97.

SaVVA, N. \& Kalliadasis, S. 2009 Two-dimensional droplet spreading over topographical substrates. Phys. Fluids 21, 092102.

SaVva, N. \& Kalliadasis, S. 2011 Dynamics of moving contact lines: A comparison between slip and precursor film models. Europhys. Lett. 94, 64004.

SAVVA, N. \& Kalliadasis, S. 2012 Influence of gravity on the spreading of two-dimensional droplets over topographical substrates. J. Engng Maths 73, 3-16. 
SaVva, N. \& Kalliadasis, S. 2013 Droplet motion on inclined heterogeneous substrates. J. Fluid Mech. 725, 462-491.

Savva, N., Kalliadasis, S. \& Pavliotis, G. A. 2010 Two-dimensional droplet spreading over random topographical substrates. Phys. Rev. Lett. 104, 084501.

Savva, N., Pavliotis, G. A. \& Kalliadasis, S. $2011 a$ Contact lines over random topographical substrates. Part 1. Statics. J. Fluid Mech. 672, 358-383.

Savva, N., Pavliotis, G. A. \& Kalliadasis, S. $2011 b$ Contact lines over random topographical substrates. Part 2. Dynamics. J. Fluid Mech. 672, 384-410.

Sibley, D. N., Nold, A., Savva, N. \& Kalliadasis, S. $2013 a$ The contact line behaviour of solid-liquid-gas diffuse-interface models. Phys. Fluids 25, 092111.

Sibley, D. N., Nold, A., Savva, N. \& Kalliadasis, S. $2013 b$ On the moving contact line singularity: Asymptotics of a diffuse-interface model. Eur. Phys. J. E 36, 26.

Sibley, D. N., SavVA, N. \& Kalliadasis, S. 2012 Slip or not slip? A methodical examination of the interface formation model using two-dimensional droplet spreading on a horizontal planar substrate as a prototype system. Phys. Fluids 24, 082105.

Smith, T. \& LindBerg, G. 1978 Effect of acoustic energy on contact angle measurements. J. Colloid Interface Sci. 66 (2), 363-366.

Strani, M. \& SABetTA, F. 1984 Free vibrations of a drop in partial contact with solid support. J. Fluid Mech. 141, 233-247.

Strani, M. \& SabetTA, F. 1988 Viscous oscillations of a supported drop in an immiscible fluid. J. Fluid Mech. 189, 397-421.

Thiele, U. \& John, K. 2010 Transport of free surface liquid films and drops by external ratchets and self-ratcheting mechanisms. Chem. Phys. 375, 578-586.

VejRazka, J., Vobecka, L. \& Tinon, J. 2013 Linear oscillations of a supported bubble or drop. Phys. Fluids 25, 062102.

Vellingiri, R., SaVva, N. \& Kalliadasis, S. 2011 Droplet spreading on chemically heterogeneous substrates. Phys. Rev. E 84, 036305.

Vukasinovic, B., Smith, M. K. \& Glezer, A. 2007 Dynamics of a sessile drop in forced vibration. J. Fluid Mech. 587, 395-423.

Wilson, S. K., Hunt, R. \& Duffy, B. R. 2000 The rate of spreading in spin coating. J. Fluid Mech. 413, 65-88. 